Boise State University

ScholarWorks

$1-1-2015$

\title{
Orthogonal Decomposition Methods to Analyze PIV, LDV, and Thermography Data of Thermally Driven Rotating Annulus Laboratory Experiments
}

Uwe Harlander

Brandenburg University of Technology

Thomas von Larcher

Freie Universität Berlin

Grady B. Wright

Boise State University

Michael Hoff

University of Leipzig

Kiril Alexandrov

Brandenburg University of Technology

See next page for additional authors 
Authors

Uwe Harlander, Thomas von Larcher, Grady B. Wright, Michael Hoff, Kiril Alexandrov, and Christoph Egbers 


\title{
Orthogonal Decomposition Methods to Analyze PIV, LDV, and Thermography Data of Thermally Driven Rotating Annulus Laboratory Experiments
}

\author{
Uwe Harlander ${ }^{1}$, Thomas von Larcher ${ }^{2}$, Grady B. Wright ${ }^{3}$, Michael Hoff ${ }^{4}$, \\ Kiril Alexandrov ${ }^{1}$, and Christoph Egbers ${ }^{1}$
}

\subsection{INTRODUCTION}

Already in the 1950s, an elegant laboratory experiment had been designed to understand how the atmospheric circulation transports heat from equatorial to polar latitudes (cf. the pioneering studies described by Hide [1958, 2010]). It consists of a cooled inner and heated outer cylinder mounted on a rotating platform, mimicking the heated tropical and cooled polar regions of Earth's atmosphere. Depending on the strength of the heating and the rate of rotation, different flow regimes had been identified in the gap: the zonal flow regime, wave regimes that can be classified by propagating waves of different wave numbers, and quasi-chaotic regimes where waves and small-scale vortices coexist.

The baroclinic annulus experiment, often called the differentially heated rotating annulus of fluid, has been accepted as a suitable laboratory model for the midlatitude large-scale flow in Earth's atmosphere. For example, Fultz [1961] and Lorenz [1964] used the heated rotating annulus as an analogy to the complex dynamics of the large-scale weather when they discussed problems related to climate variability.

Obviously, large-scale environmental flows and the flows observed in the rotating annulus show agreement

\footnotetext{
${ }^{1}$ Department of Aerodynamics and Fluid Mechanics, Brandenburg University of Technology (BTU) Cottbus-Senftenberg, Germany.

${ }^{2}$ Institute for Mathematics, Freie Universität Berlin, Berlin, Germany.

${ }^{3}$ Department of Mathematics, Boise State University, Boise, Idaho, United States of America.

${ }^{4}$ Leipzig Institute for Meteorology, University of Leipzig, Leipzig, Germany.
}

on fundamental features. A large part of this agreement is owed to the baroclinic instability mechanisms that govern atmospheric and laboratory flows [Pierrehumbert and Swanson, 1995]. This fact makes the heated rotating annulus an inspiring experiment for the community of geophysical fluid dynamics, even in the computer age.

Baroclinic instability has been investigated in the annulus not only in numerous experimental studies but also theoretically [Lorenz, 1962] and numerically [Miller and Gall, 1983; Lewis and Nagata, 2004; Randriamampianina et al., 2006; von Larcher et al., 2013; von Larcher and Dörnbrack, 2014]. The two references mentioned last are discussed in more detail in chapters 2 and 16 of the present book.

Due to its relative simple geometry as well as to the well-definable forcing parameters, the rotating annulus is still of particular interest not only for research with respect to atmospheric sciences [Gyüre et al., 2007; Ravela et al., 2010] but also in the development of computational fluid dynamics (CFD) models where the annulus data can be used as reference for the validation of new numerical concepts. In this context it is worth noting that the rotating annulus experiment described here is a reference experiment within the German priority program Multiple Scales in Fluid Mechanics and Meteorology (MetStröm) that focuses on the development of model- as well as grid-adaptive numerical simulation concepts in multidisciplinary projects (see http://metstroem.mi.fu-berlin.de).

The flow regime that develops in the cylindrical gap of the annulus depends on the radial temperature gradient between the inner and the outer cylinder, $\Delta T$, and on the rotation rate of the apparatus, $\Omega$. Thus, a $2 \mathrm{D}$ parameter space (called regime diagram) spanned by the Taylor number $\left(\propto \Omega^{2}\right)$ and by the thermal Rossby number

Modeling Atmospheric and Oceanic Flows: Insights from Laboratory Experiments and Numerical Simulations, First Edition. Edited by Thomas von Larcher and Paul D. Williams.

(C) 2015 American Geophysical Union. Published 2015 by John Wiley \& Sons, Inc. 
$\left(\propto\left(\Delta T \Omega^{-2}\right)\right)$ includes all flow regimes. Such regime diagrams are the basis of many studies and they have been experimentally derived already by Fowlis and Hide [1965] and have been refined later by other authors e.g. [Früh and Read, 1997; von Larcher and Egbers, 2005a]. The range of azimuthal wave numbers $m$ is restricted by the dimensions of the gap. Hide and Mason [1970] found an empirical law for the minimum and maximum wave number, $m_{\min } \leq$ $m \leq m_{\max }$, known as the Hide criterion reading,

$$
\frac{\pi}{4} \frac{b+a}{b-a} \leq m \leq \frac{3 \pi}{4} \frac{b+a}{b-a},
$$

with $a(b)$ as the inner (outer) radius of the gap.

One of the most fascinating aspects of the differentially heated rotating annulus is its rich time-dependent flow behavior. It is therefore not surprising that many studies have focused on this aspect. A phenomenon that has attracted much attention over many years is the so-called amplitude and structural vacillation, which is a modulation of the amplitude and the wave shape in distinct subregions of the regime diagram mentioned above. Wave dispersion and structural vacillation have been observed by e.g. Pfeffer and Fowlis [1968] using streak photographs and by Harlander et al. [2011] by particle image velocimetry (PIV). They showed the simultaneous presence of two subsequent wave modes and argued that some part of the vacillation might result from the different phase speeds of the two modes (see also Yang [1990]).

However, wave dispersion cannot explain the existence of multiple wave modes during a traverse of the regular wave regime. Therefore, Lindzen et al. [1982] numerically investigated a nonlinear version of Eady's baroclinic instability problem for the annulus, and Barcilon and Drazin [1984] investigated the problem by asymptotic techniques. In both studies, regions in the regime diagram could be identified where two modes with the same wave number may grow. Later, Früh [1996] and Früh and Read [1997] suggested that resonant wave triads are responsible for certain amplitude vacillations (see also the review on amplitude vacillations in this book in chapter 3). Such triads, besides the dominant mode, involve two other, weaker modes. Energy is redistributed between the members of a triad, and the dominant pattern vacillates with a characteristic time. Geostrophic turbulence, i.e., the irregular flow regime, is generally found at high rotation rates [Morita and Uryu, 1989; Read et al., 1992; Pfeffer et al., 1997].

The nonlinear behavior of the annulus flow motivated a number of contributions using nonlinear time series analysis to better understand the physical mechanisms. Read et al. [1992] and Früh and Read [1997], for example, used time series of temperature from probes in the fluid interior. In contrast, Sitte and Egbers [2000] and von Larcher and Egbers [2005a] used velocity time series that have been acquired by the optical laser Doppler velocimetry (LDV).

On the other hand, also linear, multivariate statistical techniques have been successfully applied to highlight certain aspects of the motion in the rotating annulus. [Read, 1993], e.g., used multivariate singular system analysis (MSSA) for phase portrait reconstructions of the annulus flow. Complex empirical orthogonal function (EOF) analysis have been applied to data from a rotating annulus with bottom topography [Pfeffer et al., 1990]. The focus of this work was to identify features of the wave propagation as a function of the Taylor number. Mundt and Hart [1994] constructed a reduced low-dimensional model of two-layer baroclinic instability by projecting the governing equations onto the EOFs of numerical flow simulations. The same should be possible by using EOFs from annulus laboratory data [Stephen et al., 1997, 1999]. Finally, Read et al. [2008] used EOFs deduced from numerical simulations to identify structural changes of the dominant modes in the annulus when the Taylor number is increased.

The differentially heated rotating annulus has also been used as a test bed for studies on weather predictability [Young and Read, 2008; Ravela et al., 2010]. Young and Read studied the breakdown of predictability for numerically deduced irregular flow regimes. In this context breeding vectors (close relatives to singular vectors) play an important role. Such vectors are an orthogonal decomposition for flows with nonorthogonal eigenmodes.

The present chapter is organized as follows. In Section 17.2 we will give details on the experimental apparatus we use and the governing nondimensional parameters. Then, in section 17.3 we will present a summary of laboratory studies on annulus flows we performed over the previous few years. In particular, we describe the multivariate orthogonal decomposition techniques we applied to the laboratory data. In Section 17.3.1 we analyze PIV and LDV data at the transition between two different wave regimes by applying the complex EOF analysis and MSSA. Subsequently, in Section 17.3.2 we analyze data from an annulus with a broken azimuthal symmetry. Similar to Pfeffer et al. [1990], we are interested in the wave propagation characteristics in a rotating annulus with "topography". The data have been retrieved simultaneously by thermography and PIV measurements. Complex EOF analysis is able to decompose the flow into features typical for the flow up- and downstream of the annulus constriction. This study was motivated by specific large-scale ocean currents like the Antarctic Circumpolar Current where the "gap width" of the flow depends on longitude. In Section 17.3.3 we decompose surface temperature data of the annulus flow in principal oscillation patterns (POPs), that is, the linear eigenmodes, and in modes of maximal growth, called singular vectors (SVs). In contrast to the traditional approach, we deduce 
these modes from the data alone without using a linear model operator. Finally, in Section 17.3.4 we decompose the annulus flow in a purely rotational and a purely divergent part. This decomposition is based on radial basis functions (RBFs) and it might prove useful in discriminating different wave types in the flow. We close the chapter with Section 17.4, where we summarize our results and provide an outlook on future work.

\subsection{EXPERIMENTAL SETUP, PARAMETERS, AND FLOW REGIMES}

\subsubsection{Setup}

Our setup (Figure 17.1), described in more detail by von Larcher and Egbers [2005b], consists of a tank with three concentric cylinders mounted on a turntable that rotates around its vertical axis of symmetry. The inner cylinder is made of anodized aluminum; the middle and outer ones are made of borosilicate glass. The temperature of the inner and outer cylinders and the rotation rate of the apparatus are controlled by the experiment software which is programmed in LabVIEW ${ }^{\circledR}$. Temperature sensors are part of the inner and outer side walls: at the inner wall at one azimuthal position in two different heights, at the outer wall at four equidistant positions in midheight

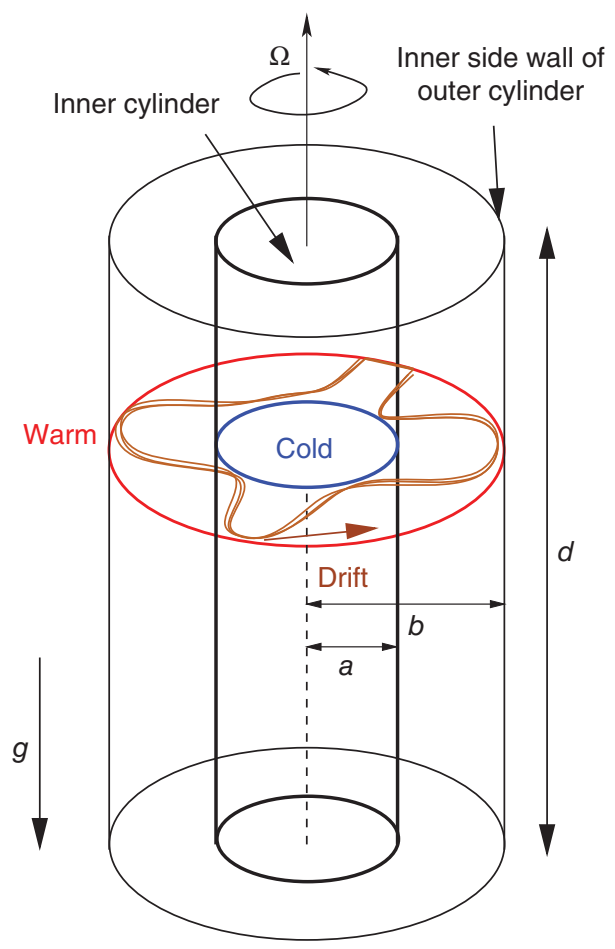

Figure 17.1. Sketch of the rotating annulus with illustration of a typical large-scale jet stream of wave number $m=4$ that has a drift relative to the rotating reference system. of the annulus. The radial temperature difference between the outer and inner walls is realized by heating the fluid in the outer annulus-shaped chamber by a heating coil and by cooling the fluid in the inner cylinder by using a thermostat. Deionized water is used as working fluid in all experiments.

All experiments were conducted in the classical $f$-plane configuration though a sloping bottom could easily be inserted and then $\beta$-plane experiments could also be performed. Furthermore, the surface is free rather than a rigid lid. We refer the reader to Fein [1973] for a comparison of experiments with a free surface and a rigid lid and to Mason [1975] for details of the influence of a sloping bottom on the flow regimes. In cases of experiments on baroclinic channel flows with narrows, a barrier was inserted in the gap, as described in Section 17.3.2.

By keeping the temperature gradient fixed but varying the rotation rate of the apparatus, sequences of regime transitions can be observed. Once the radial temperature gradient is settled, the spin-up time is found to be less than 30 min for steady waves and up to 40 min for complex flows. Observations were usually done up to several hours per parameter point, extended partly in complex flow regimes.

\subsubsection{Parameters}

The shape of the annulus is defined by the radius ratio $\eta$ and the aspect ratio $\Gamma$ with

$$
\eta=\frac{a}{b}, \quad \Gamma=\frac{d}{b-a},
$$

where the inner radius $a=45 \mathrm{~mm}$, the outer radius $b=120 \mathrm{~mm}$, and the fluid depth $d=135 \mathrm{~mm}$ (implying $\eta=0.38$ and $\Gamma=1.8$ for our apparatus).

Beyond these geometric parameters, the fluid motion is governed by the two dynamic control parameters, the rotation rate of the annulus, $\Omega$, and the radial temperature difference in the cylindrical gap, $\Delta T$. These parameters determine the nondimensional Taylor number Ta and thermal Rossby number Ro, as already mentioned above. The two numbers read

$$
\mathrm{Ta}=\frac{4 \Omega^{2}(b-a)^{5}}{v^{2} d}, \quad \text { Ro }=\frac{g d \alpha \Delta T}{\Omega^{2}(b-a)^{2}},
$$

where $v$ is the kinematic viscosity, $g$ the acceleration due to gravity, and $\alpha$ the volumetric expansion coefficient. The Taylor number measures the rotation rate with respect to the viscous effect, and the thermal Rossby number corresponds to the ratio of buoyancy and Coriolis terms and therefore indicates a thermal stratification of the flow. 
Another important parameter is the Prandtl number, defined by

$$
\operatorname{Pr}=\frac{v}{\kappa}
$$

where $\kappa$ is thermal conductivity and $\operatorname{Pr}$ describes the physical properties of the fluid, with $\mathrm{Pr}=7.16$ as the appropriate value for water at $20^{\circ} \mathrm{C}$ (see chapter 2 this volume and Fowlis and Hide [1965] for more details on the effect of the Prandtl number).

\subsubsection{Flow regimes}

By keeping $\Delta T=7.5 \mathrm{~K}$ constant and varying $\Omega$ in the range $3 \times 10^{6}<\mathrm{Ta}<3 \times 10^{8}$, the regime diagram typically shows specific flow regimes characterized by the azimuthal wave number $0 \leq m \leq 4$, i.e., the axisymmetric $m=0$ basic flow at $3 \times 10^{6}<$ Ta $<6 \times 10^{6}$, the wave flow regime with $2 \leq m \leq 3$ at about $6 \times 10^{6}<$ $\mathrm{Ta}<1.5 \times 10^{8}$, and the regime with structural vacillations (called irregular wave regime when the wave structure is deformed in an irregular way) with dominant wave $m=4$ at higher Taylor numbers (see Figure 17.2). Note, that $m_{\max }=4$ is in good agreement with the Hide criterion (eq. 17.1). Hysteresis occurs when one can find different regimes for the same control parameters where the parameters have been approached from different directions that is an increasing or decreasing Taylor number. Hysteresis is a well-known phenomenon for annulus experiments and can generically be observed in free-surface [Sitte and Egbers, 2000; von Larcher and Egbers, 2005b] and rigid lid experiments [Cole, 1971; Hignett et al., 1985]. For our experimental setup a rather broad region of hysteresis exists at the transition between $m=3$ and $m=4$. The existence of hysteresis depends not only on Ta, Ro, and $\mathrm{Pr}$ but also on $\eta$ and $\Gamma$ [von Larcher and Egbers, 2005b].

Moreover, complex vacillating flow patterns are found in the transition from axisymmetric flow to the wavy flow regime (see Figure 17.3, upper row), where mode

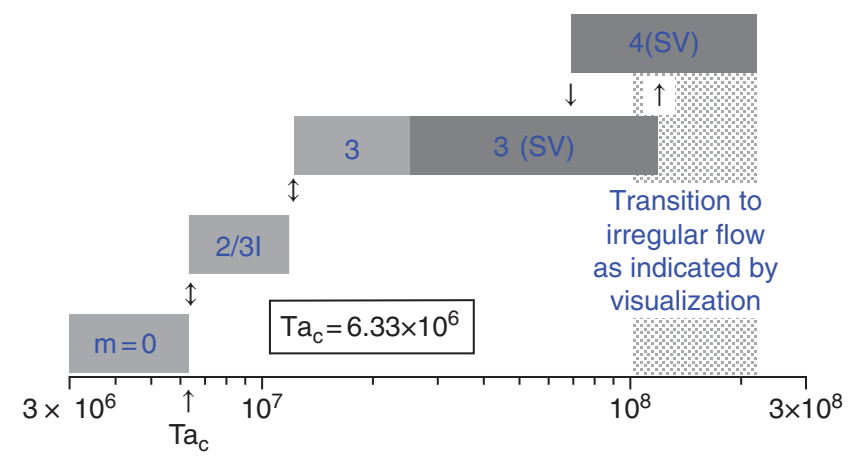

Taylor number Ta

Figure 17.2. Regime diagram observed for $\eta=0.38, \Gamma=1.8$, and $\Delta T=7.5 \mathrm{~K}$ [von Larcher and Egbers, 2005b]. interaction between mode $m=2$ and $m=3$ occurs. Furthermore, structural (or shape) vacillations are found at high Taylor numbers (Figure 17.3, lower row), where cold cells separate from the inner wall, move radially outward, and then return to the inner cylinder while their outer boundary largely remains unaffected.

While the complex flow patterns in the first transition zone might be identified as a superposition of two coexisting waves with different zonal wave numbers and phase speeds, denoted as interference vacillation (IV), which was found to occur in experiments with a free surface [Pfeffer and Fowlis, 1968; Kaiser, 1970; Harlander et al., 2011] as in rigid-lid experiments [Früh and Read, 1997], the structural vacillations observed at higher Taylor numbers could be consistent with an oscillation of a higher radial mode of the same azimuthal wave as observed in experiments by Pfeffer et al. [1980] and described theoretically by Weng et al. [1986].

\subsection{RESULTS}

Multivariate statistical techniques are suitable to understand better the variability of the heated rotating flow. We consider here four different methods: EOF analysis, MSSA, POP analysis, and SV analysis. It is worth to briefly mention the field of application of the different techniques. This enables the reader to assess which technique is good for what purpose. For many applications it is useful to decompose noisy multivariate data sets into subsets or subspaces. A few patterns might span the "signal subspace" where the noise is captured in the "noise subspace." The specification of relevant patterns can be done in many different ways, ranging from eigenmodes (e.g., in terms of Bessel and trigonometric functions for cylindrical geometry) to patterns that optimize certain statistical moments [H. von Storch, 1995].

EOFs are defined as those patterns that are powerful in explaining variance and thus the EOF method is the method of choice for analyzing the variability of fields. It is therefore widely used in the geosciences [Lorenz, 1956; von Storch and Zwiers, 1999]. This method also goes by different names, e.g., principal component analysis or proper orthogonal decomposition. The EOF method is able to find the spatial patterns of variability and their time variation and provides a measure for the relevance of each pattern. Simply speaking, the EOF method breaks the data into modes of variability that might (as is the case for our data) be interpreted as physical modes of the system. Strikingly, it can be shown that the EOF method provides the most efficient way of capturing the dominant components of a high-dimensional process with often surprisingly few modes [Holmes et al., 1996]. However, more appropriate in our context is the use of the complex EOF (CEOF) analysis [Pfeffer et al., 1990; von Storch 


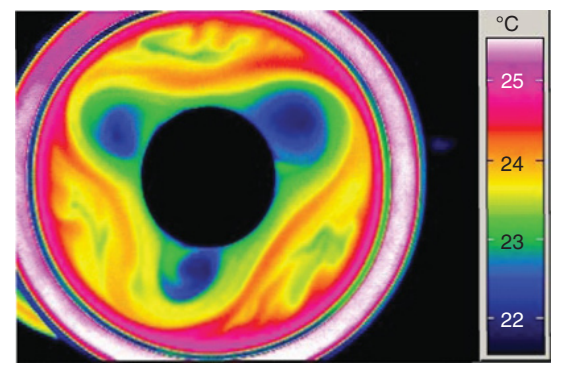

$t=0 \mathrm{~s}$

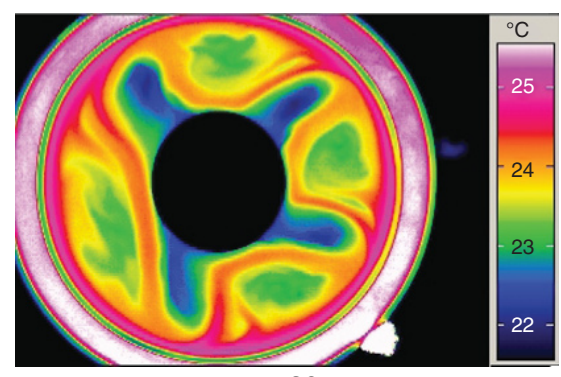

$t=+30 \mathrm{~s}$

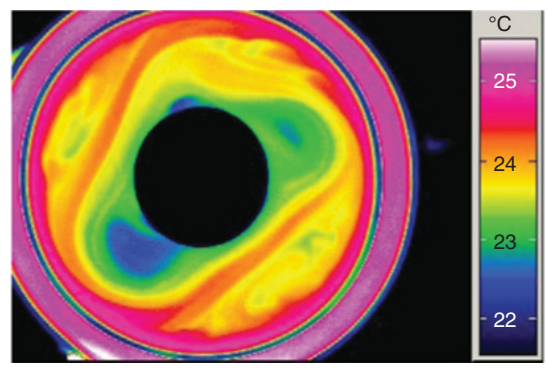

$t=+1200 \mathrm{~s}$

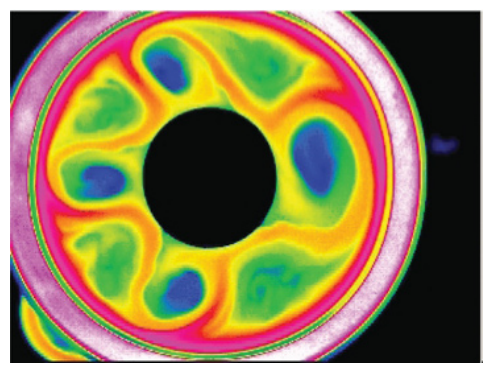

$t=+60 \mathrm{~s}$

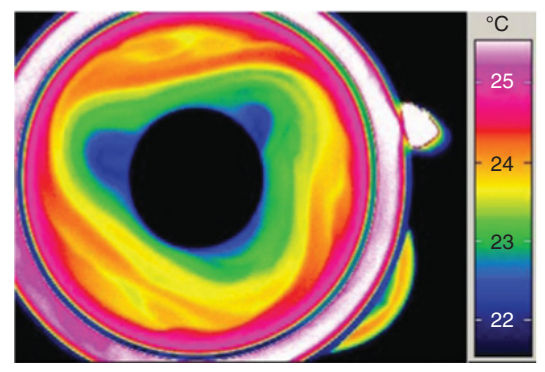

$t=+3000 \mathrm{~s}$

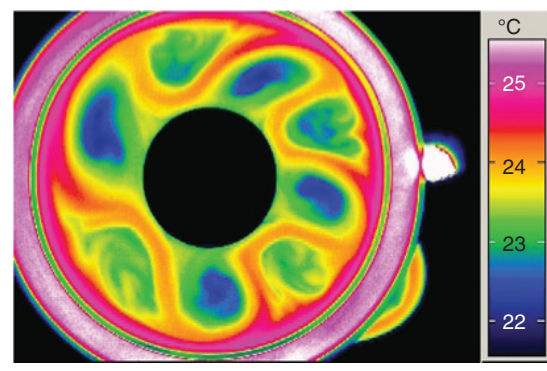

$t=+90 \mathrm{~s}$

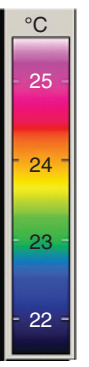
Figure 17.3. Sequence of thermographic measurements describing vacillating flows [von Larcher and Egbers, 2005b]. Top: Wave
mode competition of wave number $m=2$ and $m=3$ at Ta $=1.08 \times 10^{7}$, Ro $=3.00$. Bottom: Structural vacillation flow, radial oscillation of a wavy flow of wave number $m=4$ at Ta $=7.65 \times 10^{7}$, Ro $=0.41$, with $t$ as relative time.

and Zwiers, 1999] that reveals propagating patterns of variability by single CEOFs, whereas pure EOFs capture only standing modes of variability.

The MSSA is a generalization of the single-time-series SSA method to multiple time series [Broomhead and King, 1986; Read, 1992; Vautard, 1995]. These time series may contain observations of a certain variable at different locations (as in our case) or even observations of different variables. In classical EOF analysis the dominant spatial patterns are captured by diagonalizing the covariance matrix. As discussed Vautard [1995], the coordinates of the state vector in the EOF analysis represent different locations in space at the same time. In an SSA, the state vector contains values at the same locations but at different time lags. CEOF analysis is a special case of the MSSA method; however, MSSA deals with more temporal degrees of freedom than spatial ones, allowing the investigation of spectral properties of the data. In contrast, CEOF contains only a single lag but a large number of spatial points. The MSSA method allows one to detect oscillating features in noisy time series where oscillations occur frequently only during certain time periods. The larger generality of the MSSA is purchased by a larger amount of computing time. A detailed description of the MSSA method is beyond the scope of the present chapter but is given by Dettinger et al. [1995], Vautard [1995], and Elsner and Tsonis [1996].

POPs are empirical, that is, data estimated normal modes [Hasselmann, 1988]. POPs are another way of decomposing a data set into a signal and noise subspace. To evaluate POPs, the system matrix corresponding to a linear model has to be found as described, e.g., by von Storch and Zwiers [1999]. Frequently, POPs correspond with EOFs, though this correspondence is not guaranteed from a mathematical point of view. Also the correspondence between true normal modes and POPs is not always obvious [J.-S. von Storch, 1995]. In real data, linearly unstable modes occur only in a nonlinearly saturated state. Thus POP modes are either neutral or damped. In contrast, a linear operator might allow for unstable modes that cannot be covered by any data based method. Nevertheless, POP analysis has proven to be useful in a broad range of applications and can be considered as one of the routine tools in climate research. From the empirically estimated system matrix, not only POPs can be computed. A further useful step is to estimate SV, from the system matrix. SVs correspond to those initial perturbations that grow in an optimal sense with respect to a chosen norm within a predefined time interval, the so-called optimization time. For large-scale baroclinic systems, SVs might play an important role and they might be even more relevant for real flows than unstable normal modes [Badger and Hoskins, 2001].

All the four mentioned orthogonal decompositions (EOF analysis, MSSA, POP analysis, SV analysis) are related via the data matrix. Let us briefly describe how. A variable $X_{i}$ is observed at $M$ different arbitrarily spaced points, $i=1,2, \ldots, M$, and at $P$ different instances of 
time. Note that $t_{j+1}=t_{j}+j \Delta$, where $\Delta$ is the sampling interval, and that the first measurement was done at time $t_{1}$ and the last at time $t_{P}=t_{N}+n \Delta$, where $n$ is an integer number. Subtracting the mean from each time series $x_{i}=X_{i}-\bar{X}$, we can put the data into a generalized $N \times M$ data matrix

$\mathbf{F}^{n \Delta}=\left(\begin{array}{cccc}x_{1}\left(t_{1}+n \Delta\right) & x_{2}\left(t_{1}+n \Delta\right) & \cdots & x_{M}\left(t_{1}+n \Delta\right) \\ x_{1}\left(t_{2}+n \Delta\right) & x_{2}\left(t_{2}+n \Delta\right) & \cdots & x_{M}\left(t_{2}+n \Delta\right) \\ \vdots & \vdots & \ddots & \vdots \\ x_{1}\left(t_{N}+n \Delta\right) & x_{2}\left(t_{N}+n \Delta\right) & \cdots & x_{M}\left(t_{N}+n \Delta\right)\end{array}\right)$

where $n \Delta, n=0,1,2, \ldots$, defines a time delay. An extended matrix $F_{m}$ can be defined as

$$
\mathbf{F}_{m}=\left(\begin{array}{llll}
\mathbf{F}^{\Delta}, & \mathbf{F}^{2 \Delta}, & \mathbf{F}^{3 \Delta}, \ldots, \mathbf{F}^{m \Delta}
\end{array}\right) .
$$

Note that $\mathbf{F}_{m}$ is an $N \times m M$ matrix formed by the $m$ submatrices $\mathbf{F}^{\Delta}, \ldots, \mathbf{F}^{m \Delta}$. EOFs are the eigenvectors of $\left(\mathbf{F}^{0 \Delta}\right)^{T} \mathbf{F}^{0 \Delta}$, space-time EOFs (MSSA modes) with time window $m$ are the eigenvectors of $\mathbf{F}_{m}^{T} \mathbf{F}_{m}$, POPs are the eigenvectors of $\mathbf{P}=\left(\left(\mathbf{F}^{1 \Delta}\right)^{T} \mathbf{F}^{0 \Delta}\right)\left(\left(\mathbf{F}^{0 \Delta}\right)^{T} \mathbf{F}^{0 \Delta}\right)^{-1}$, and singular vectors with optimization time $\Delta$ and based on the Euclidean norm are the eigenvectors of $\mathbf{P}^{T} \mathbf{P}$. All these eigenvectors form an orthogonal basis. Therefore, the data vector at time $i$ can be written as

$$
\vec{F}=\sum_{j} a\left(t_{i}\right)_{j} \vec{\Phi}_{j}
$$

where $\vec{\Phi}_{j}$ is the $j$ th eigenvector from an EOF, MSSA, POP, or SV analysis, and the coefficients $a\left(t_{i}\right)_{j}$ are found by a suitable projection of the eigenvectors on the data vector.

In Section 17.3.4 we discuss a novel orthogonal decomposition that is not premised on a statistical basis. Here we use a mesh-free data reconstruction method that is based on RBFs. Using these basis functions, we can decompose the horizontal velocity data into a sum of divergence-free and curl-free parts. Such a decomposition can be very useful in discriminating different wavefields in the annulus.

\subsubsection{EOF and MSSA Analysis of Wave Interactions With PIV and LDV Measurements}

In this section, we present the analysis of velocity data from classical $f$-plane thermally driven rotating annulus experiments with $\eta=0.38, \Gamma=1.8$, and $\operatorname{Pr}=7.16$ (see (17.2) and (17.4)), recovered by PIV and LDV (see Harlander et al. [2011] for details). The following questions will be addressed: (i) Can the statistical analysis detect coexisting wave modes during a traverse between two regular wave regimes? (ii) Can we find coexisting modes in the transition region to the quasi-chaotic regime or is the flow dominated by random fluctuations?

The data we used are sampled twice (PIV) or 20 times (LDV) per revolution of the annulus and cover time periods sufficiently long to allow for the application of multivariate statistical techniques. While the PIV data consist of the horizontal flow field, the LDV data consist of 20 time series regularly distributed along a circle in the annulus at mid-radius, i.e. $(a+b) / 2$ (see Figure 17.1). Note that the sampling rate of the LDV measurement is 10 times larger than the one of the PIV measurements. However, with respect to a fixed spatial point, the LDV samples the data just once per revolution. Still, short-lived structures typical for more transient flows are better resolved in the LDV data.

The PIV system is used to measure the horizontal velocity components $15 \mathrm{~mm}$ below the fluid surface. Each experiment lasts typically $50 \tau$, where $\tau$ is the revolution period of the annulus. We sample the PIV data with $\tau / 2$, i.e. two observations per revolution. The PIV camera is mounted in an inertial frame above the cylinder, that is, the camera does not corotate with the cylinder. To obtain the velocity components in the corotating frame, we subtract the solid-body velocity $\vec{v}=\vec{\Omega} \times \vec{r}$ from each observed PIV velocity rigid-field. A preprocessing of the data is needed to eliminate erroneous vectors and to homogenize the data.

The radial velocity component is measured with the LDV that was fixed in the inertial frame, too. The measurements take place $2 \mathrm{~mm}$ below the fluid surface at midradius of the annulus. The large data set is reduced by an appropriate averaging. Furthermore, linear interpolation is applied to obtain a homogeneous data set with regular grid distance $\Delta \Phi=18^{\circ}$ and $\Delta t=\tau / 20$. These preprocessed LDV data are then analyzed using the MSSA software toolkit by Dettinger et al. [1995].

The MSSA software package is particularly suited to detect (intermittent) oscillations in noisy time series as well as in multivariate data. With regard to the aims of our study, this makes the method particularly suitable to find structures in the transition region to the quasichaotic regime where the waves become more and more irregular. In the specific experimental setup used here, this regime occurs when the Taylor number is larger than $10^{8}$ and the thermal Rossby number is smaller than 0.5 (cf. Figure 17.2).

Owing to the fixed PIV camera, the errors of PIV observations grow with growing angular velocity of the cylinder. In contrast, using LDV, the radial velocity component can be observed even for large angular velocities of the annulus with high accuracy. Thus, LDV data from the irregular wave regime will be analyzed by using the MSSA method (question (ii)). Instead, the preprocessed PIV data were analyzed by using the CEOF method with the focus on question (i).

A detailed description of our approach to find coupled propagating patterns with the $\mathrm{CEOF}$ is given by Harlander et al. [2011]. Briefly speaking, we use the Hilbert 
transform method to make the (horizontal) velocity components $(u, v)$ complex and then formed extended time series by combining the two complex time series. With this new time series we built the covariance matrix and computed its eigenvectors (i.e., the CEOFs) and the corresponding time-dependent coefficients.

17.3.1.1. Analysis of PIV Measurements. As described, PIV measurements were performed to detect complex flows during the traverse between two regular wave regimes. The measurements presented here have been conducted at $\mathrm{Ta}=1.74 \times 10^{7}, \mathrm{Ro}=1.30$ (i.e., $\Omega=0.50 \mathrm{rad} / \mathrm{s}, \Delta T=6.7 \mathrm{~K}$ ). That parameter point is close to the transition from the steady wave regime of wave number $m=3$ to the structural vacillation (SV) regime, i.e., $m=3$ (SV) (cf. Figure 17.2, but note that here $\Delta T=7.5 \mathrm{~K}$ and that for larger $\Delta T$ transitions occur at larger Ta).

The eigenvalue spectrum (not shown) is dominated by the first eigenvalue that contains more than $40 \%$ of the total variance of the flow, and the second eigenvalue includes about $10 \%$ of the total variance. It should be noted that the variance distribution depends on the data quality and on Ro and Ta. The lower row of Figure 17.4 shows the corresponding real part of the CEOFs and the upper row their time evolution. Note that, in general, the real and imaginary parts of the CEOFs and their time series show a $90^{\circ}$ phase difference [von Storch and Zwiers, 1999].

The velocity field can be reconstructed via (17.5). CEOF1 together with the time-dependent coefficient determines a prograde propagating wave (i.e., a wave propagating in the direction of the annulus revolution) with wave number $m=3$; CEOF2, in contrast, determines a rather regular and slowly retrograde wave (propagating in the opposite direction of the annulus revolution) with wave number $m=4$. By combining the first two patterns (which then contain about $50 \%$ of the total variance), a wavy jet flow with dominant wave number $m=3$ is found which shows slow vacillations due to an interference with the (weak) mode pattern with $m=4$. Further details and also patterns for different Ta and Ro are discussed in Harlander et al. [2011].

From the time-dependent coefficients (Figure 17.4, top panel), the drift rates of the dominant mode $m=3$ and of the weak mode $m=4$ are found to be 0.021 and $-0.007 \mathrm{rad} / \mathrm{s}$, respectively. Slow retrograde propagating modes are rather exceptional but have been reported earlier [Früh and Read, 1997]. It appears that the propagation of the weak mode is strongly affected by the dominant mode of the system and that linear wave theory fails to describe its anomalous retrograde propagation, as all unstable baroclinic modes should propagate with the volume-averaged mean flow (which was estimated to be approximately $0.009 \mathrm{rad} / \mathrm{s}$ ), according to the linear Eady model.

To summarize the main results of the CEOF analysis, we find that in the rather stable $m=3$ regime the presence of higher modes and their linear interaction with the leading wave mode can give rise to slow modulations. However, the existence of the $m=4$ mode in the $m=3$ wave regime cannot be explained by linear theory.

17.3.1.2. Analysis of $L D V$ Measurements. Next we discuss the results from the MSSA of the LDV data. As mentioned above, a strong feature of the MSSA is its ability to detect oscillating/propagating features in noisy data. Thus, the MSSA seems to be more suitable than EOF analysis to find excited propagating modes in flow with structural vacillations and "irregular wave regimes." Note that we call a wave irregular when it shows significant transient features. In contrast, for a turbulent flow regime a dominant wave can no longer be observed. Here we apply the MSSA to a parameter point in the irregular flow regime, i.e., at Ta $=3.76 \times 10^{8}$, Ro $=0.14$ $(\Omega=2.32 \mathrm{rad} / \mathrm{s}, \Delta T=6.9 \mathrm{~K})$.

Figure 17.5 (top panel) shows the preprocessed LDV data. The data are presented in the form of a space-time diagram, where the abscissa runs from 0 to $2 \pi$, covering the spatial structure of the radial velocity at midradius of the annulus. Although the flow is much more noisy than for the PIV experiment, we clearly can identify a wave pattern with $m=4$ that propagates prograde with a phase speed of $0.011 \mathrm{rad} / \mathrm{s}$.

The eigenvalue spectrum is broad (Figure 17.5, upper right part). Noise is usually part of the flat tail in the eigenvalue spectrum. Here, we define the noise level to be at $1 \%$ of the total variance. Note that this is a qualitative measure, and it is not the exact signal-to-noise level of our experiments. The first two eigenvalues explain $36 \%$ of the total variance and the next two eigenvalues are also clearly above the defined noise level.

Similar to the description of flow field reconstruction using the CEOF method, reconstructing the data by using just the first two space-time EOFs (ST-EOFs) gives a filtered version of the original data (Figure 17.5, bottom left). Instructive is the reconstruction by ST-EOF 3 and 4, explaining at least $5 \%$ of the total variance (Figure 17.5, bottom right). The reconstruction reveals a wave pattern with wave number $m=5$ that propagates essentially with the same phase speed as the dominant $m=4$ wave. Finally, note that the $m=5$ wave pattern shows slight amplitude vacillations. Moreover, its phase speed is less constant than the phase speed of the dominant wave.

Roughly speaking, for the irregular flow regime, the first wave modes seem to be less dispersive than for the 

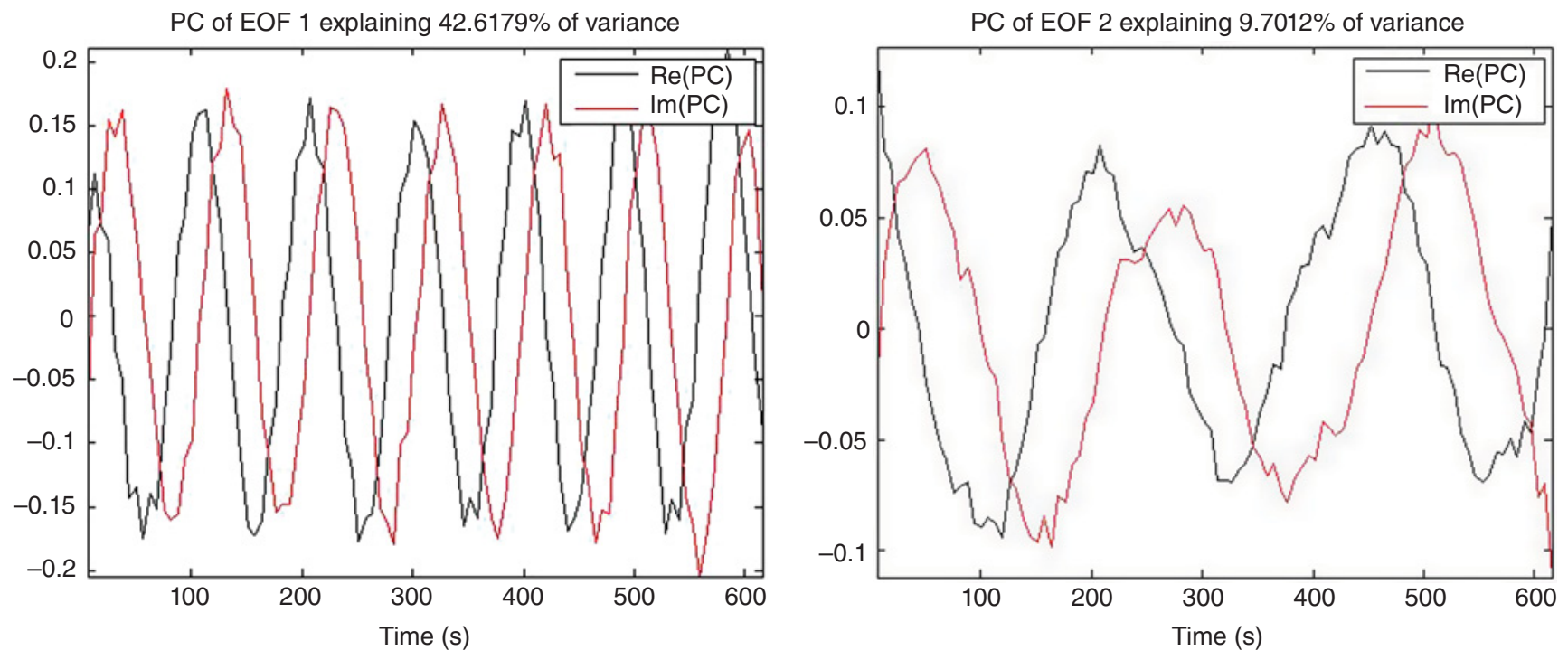

Real EOF1 42.6179\%
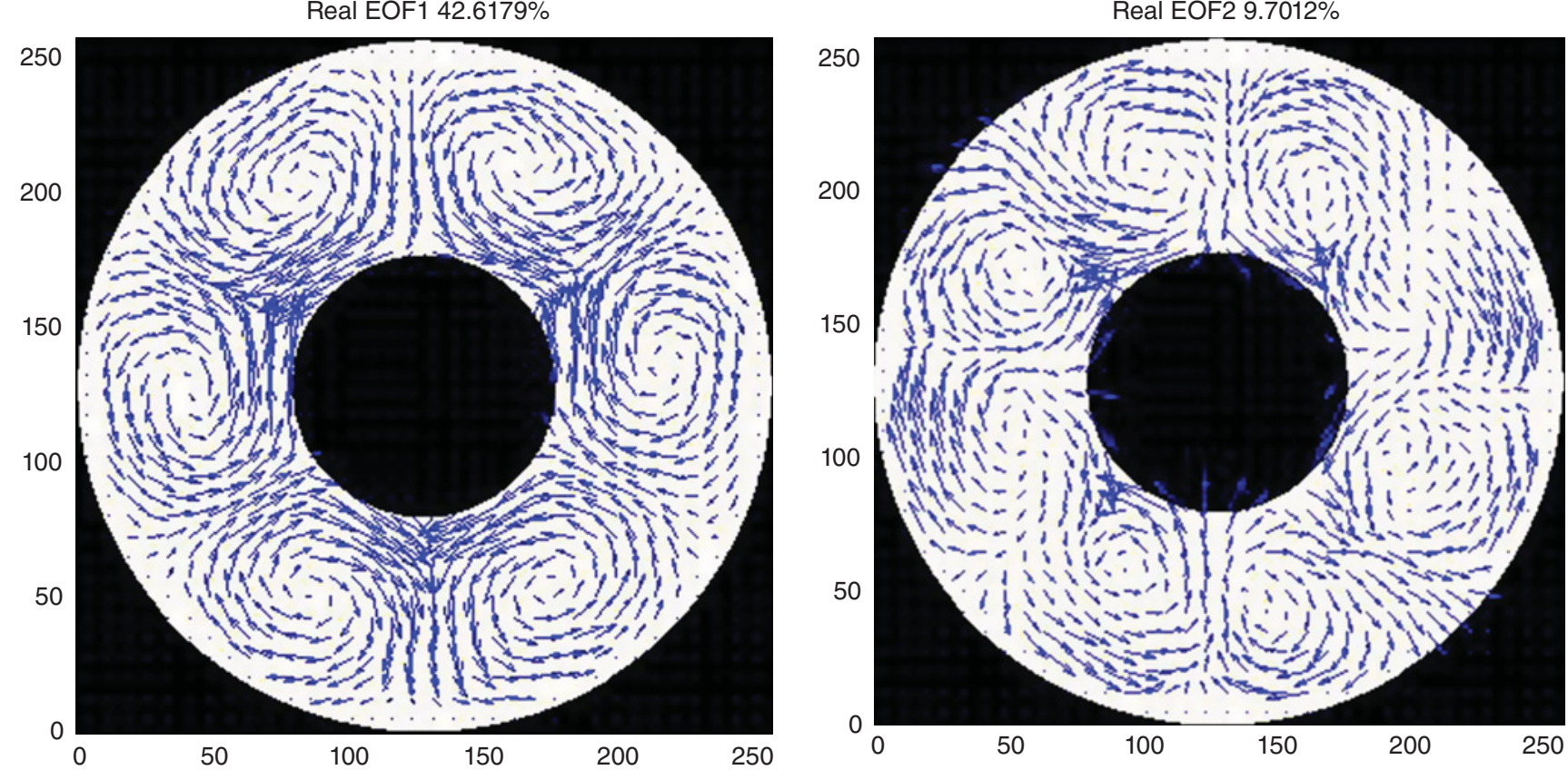

Figure 17.4. CEOF analysis of PIV measurements (given in corotating frame). Top: The principal component (PC) of CEOF1 (real part black, imaginary part red) (left) and of CEOF2 (right). Bottom: Real part of CEOF1 (left) and of CEOF2 (right). The imaginary parts of the two CEOFs are phase-shifted versions of the real parts.

vacillating wave regime discussed above. Classical Eady modes are nondispersive, and we might conclude that the first four EOFs do not resolve the nonlinear features of the wave modes in the irregular flow. Hence, EOFs from the noisy part of the spectrum need to be considered to address the irregularity of large Taylor/small Rossby number regimes.

Finally, in Figure 17.6 and Table 17.1 we show the variance distribution (in percent of the total variance) for the first 12 wave modes $m=1,2, \ldots, 12$ along a transection through the regime diagram, from the azimuthal flow regime ( $m=0)$ to the slightly irregular $m=4$ wave regime $\left(6.79 \times 10^{6} \leq \mathrm{Ta} \leq 4.77 \times 10^{8}, 5.73 \times 10^{-2} \leq \mathrm{Ro} \leq 4.0\right.$, $\Delta T=8 \mathrm{~K})$. The transition from $m=0$ to $m=2$ occurs at $(\mathrm{Ta}, \mathrm{Ro})=\left(9.49 \times 10^{6}, 2.82\right)$. A dominant wave with $m=2$ establishes, but other waves $(m=3, \ldots, 6)$ are also present in the variance spectrum. This indicates that the flow with zonal wave number 2 exhibits some vacillations. At $(\mathrm{Ta}, \mathrm{Ro})=\left(2.3 \times 10^{7}, 1.19\right)$, the flow regime changes to $m=3$ and we can find this wave and its harmonics 

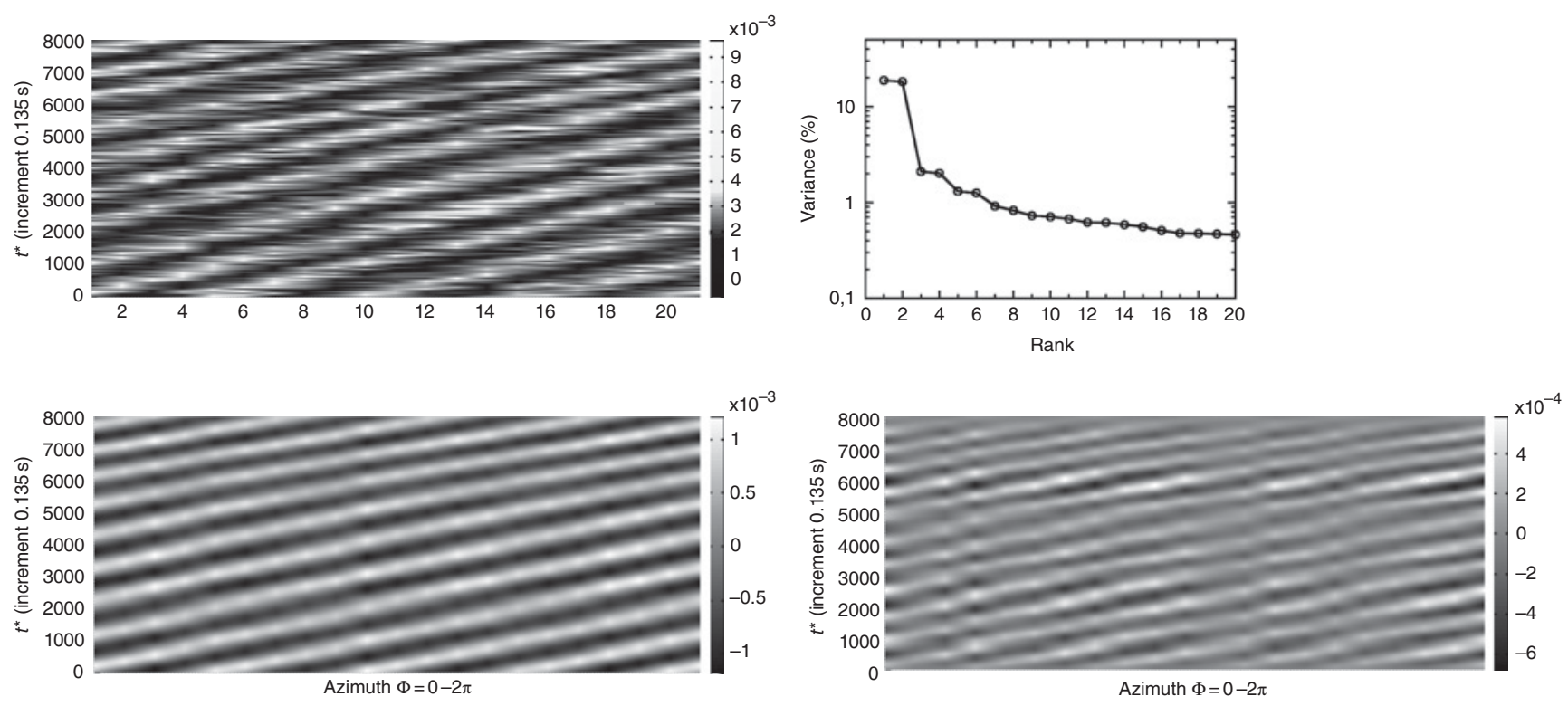

Figure 17.5. MSSA analysis of a wave mode of dominant wave number $m=4$. Upper left: Space-time plot of the homogenized LDV data set, radial velocity $[\mathrm{m} / \mathrm{s}]$ over azimuth $0-2 \pi$. Upper right: The first 20 eigenvalues of the MSSA covariance spectrum. Note that a logarithmic scale has been used. Lower left: Reconstructed space-time plot using the first two MSSA eigenvectors. Lower right: Reconstructed space-time plot using the third and the fourth MSSA eigenvectors.

(a)

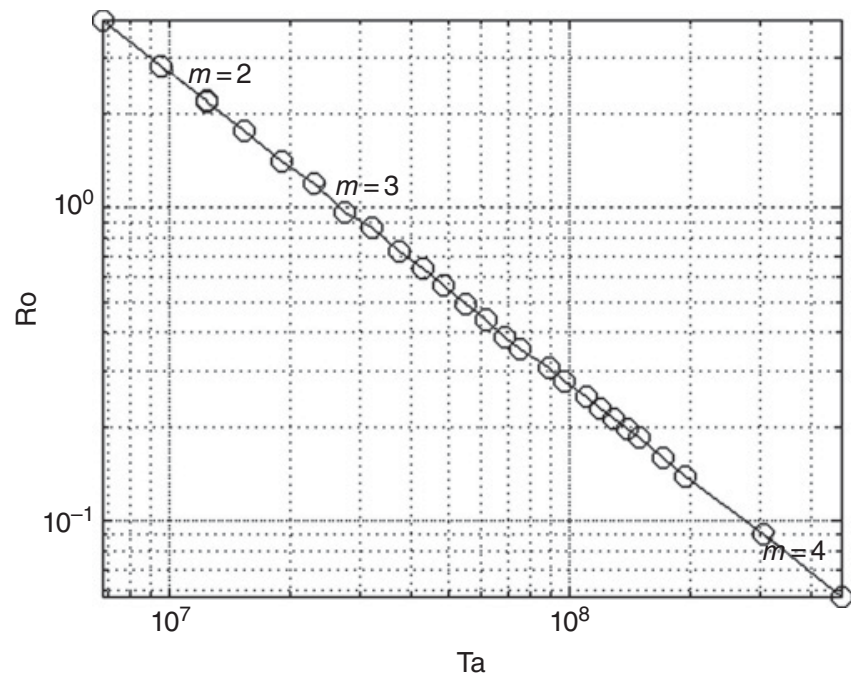

(b)

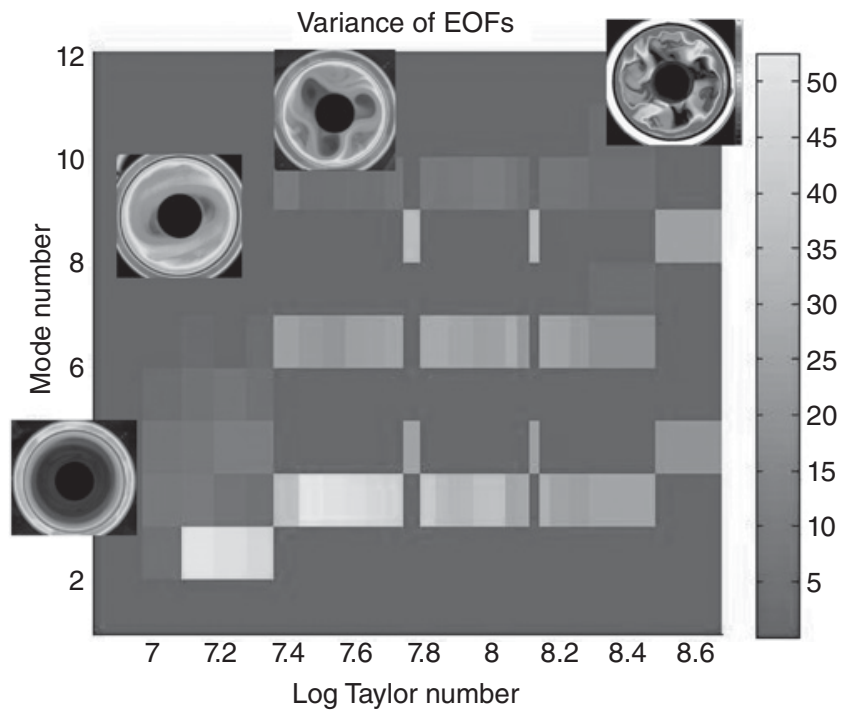

Figure 17.6. EOF variance spectra obtained along a transection through the wave regime. Left: Each circle in the Ta Rodiagram corresponds with an experiment. Right: Distribution of the variance (in \% of the total variance) for the first 12 wave modes as a function of Ta. See also Table 17.1. For color detail, please see color plate section.

$(m=6,9)$ in the EOF variance spectrum. When we increase Ta, the dominant wave becomes weaker and the first harmonics stronger. At the transition to $m=4$ (at $\left.(\mathrm{Ta}, \mathrm{Ro})=\left(3.04 \times 10^{8}, 9.01 \times 10^{-2}\right)\right)$ the first harmonic comprises as much variance as the $m=4$ mode. The flow is rather irregular and it becomes more and more difficult to identify a dominant wave. The EOF variance spectrum starts to become broader. Within the $m=3$ regime we find transitions to the $m=4$ flow indicating regions of multiple equilibria. Note that the irregular wave flow must not be confused with turbulent flow. For the first a wave pattern is still present whereas for the latter it is not. 
Table 17.1. Variance in \% explained by the zonal wave modes $m=1,2, \ldots, 12$.

\begin{tabular}{|c|c|c|c|c|c|c|c|c|c|c|c|c|c|}
\hline $\mathrm{Ta}$ & Ro & 1 & 2 & 3 & 4 & 5 & 6 & 7 & 8 & 9 & 10 & 11 & 12 \\
\hline $6.79 \mathrm{E}+06$ & $4.00 \mathrm{E}+00$ & 0.0 & 0.0 & 0.0 & 0.0 & 0.0 & 0.0 & 0.0 & 0.0 & 0.0 & 0.0 & 0.0 & 0.0 \\
\hline $9.49 \mathrm{E}+06$ & $2.82 \mathrm{E}+00$ & 0.0 & 4.8 & 8.7 & 7.1 & 3.1 & 0.0 & 0.0 & 0.0 & 0.0 & 0.0 & 0.0 & 0.0 \\
\hline $1.24 \mathrm{E}+07$ & $2.21 \mathrm{E}+00$ & 0.0 & 37.0 & 6.8 & 5.8 & 4.1 & 0.7 & 0.0 & 0.0 & 0.0 & 0.0 & 0.0 & 0.0 \\
\hline $1.24 \mathrm{E}+07$ & $2.19 \mathrm{E}+00$ & 0.0 & 51.1 & 9.1 & 8.2 & 5.7 & 1.9 & 0.0 & 0.0 & 0.0 & 0.0 & 0.0 & 0.0 \\
\hline $1.54 \mathrm{E}+07$ & $1.76 \mathrm{E}+00$ & 0.0 & 48.9 & 6.3 & 11.5 & 6.8 & 0.0 & 0.0 & 0.0 & 0.0 & 0.0 & 0.0 & 0.0 \\
\hline $1.91 \mathrm{E}+07$ & $1.40 \mathrm{E}+00$ & 0.0 & 46.0 & 4.3 & 10.7 & 4.9 & 2.9 & 0.0 & 0.0 & 0.0 & 0.0 & 0.0 & 0.0 \\
\hline $2.30 \mathrm{E}+07$ & $1.19 \mathrm{E}+00$ & 0.0 & 0.0 & 36.5 & 0.0 & 0.0 & 24.7 & 0.0 & 0.0 & 9.6 & 0.0 & 0.0 & 0.0 \\
\hline $2.74 \mathrm{E}+07$ & 9.67E-01 & 0.0 & 0.0 & 52.6 & 0.0 & 0.0 & 23.0 & 0.0 & 0.0 & 3.4 & 0.0 & 0.0 & 0.0 \\
\hline $3.20 \mathrm{E}+07$ & 8.64E-01 & 0.0 & 0.0 & 50.0 & 0.0 & 0.0 & 21.3 & 0.0 & 0.0 & 4.5 & 0.0 & 0.0 & 0.0 \\
\hline $3.75 \mathrm{E}+07$ & 7.24E-01 & 0.0 & 0.0 & 48.1 & 0.0 & 0.0 & 24.0 & 0.0 & 0.0 & 5.3 & 0.0 & 0.0 & 0.0 \\
\hline $4.29 E+07$ & $6.40 \mathrm{E}-01$ & 0.0 & 0.0 & 45.6 & 0.0 & 0.0 & 24.9 & 0.0 & 0.0 & 4.5 & 0.0 & 0.0 & 0.0 \\
\hline $4.85 \mathrm{E}+07$ & $5.62 \mathrm{E}-01$ & 0.0 & 0.0 & 44.1 & 0.0 & 0.0 & 26.8 & 0.0 & 0.0 & 7.3 & 0.0 & 0.0 & 0.0 \\
\hline $5.50 \mathrm{E}+07$ & 4.89E-01 & 0.0 & 0.0 & 0.0 & 25.7 & 0.0 & 0.0 & 0.0 & 36.1 & 0.0 & 0.0 & 0.0 & 6.1 \\
\hline $6.17 \mathrm{E}+07$ & 4.40E-01 & 0.0 & 0.0 & 41.4 & 0.0 & 0.0 & 28.4 & 0.0 & 0.0 & 8.4 & 0.0 & 0.0 & 0.0 \\
\hline $6.88 \mathrm{E}+07$ & $3.85 \mathrm{E}-01$ & 0.0 & 0.0 & 34.6 & 0.0 & 0.0 & 28.4 & 0.0 & 0.0 & 7.7 & 0.0 & 0.0 & 0.0 \\
\hline $7.56 \mathrm{E}+07$ & $3.54 \mathrm{E}-01$ & 0.0 & 0.0 & 33.1 & 0.0 & 0.0 & 27.1 & 0.0 & 0.0 & 8.5 & 0.0 & 0.0 & 0.0 \\
\hline $8.89 \mathrm{E}+07$ & $3.08 \mathrm{E}-01$ & 0.0 & 0.0 & 37.4 & 0.0 & 0.0 & 24.1 & 0.0 & 0.0 & 10.3 & 0.0 & 0.0 & 0.0 \\
\hline $9.73 \mathrm{E}+07$ & 2.80E-01 & 0.0 & 0.0 & 37.5 & 60.0 & 0.0 & 25.3 & 0.0 & 0.0 & 9.9 & 0.0 & 0.0 & 0.0 \\
\hline 1.10E +08 & $2.51 \mathrm{E}-01$ & 0.0 & 0.0 & 26.9 & 0.0 & 0.0 & 29.1 & 0.0 & 0.0 & 7.9 & 0.0 & 0.0 & 0.0 \\
\hline $1.19 \mathrm{E}+08$ & 2.30E-01 & 0.0 & 0.0 & 27.2 & 0.0 & 0.0 & 21.6 & 0.0 & 0.0 & 5.0 & 0.0 & 0.0 & 0.0 \\
\hline $1.29 \mathrm{E}+08$ & $2.11 \mathrm{E}-01$ & 0.0 & 0.0 & 0.0 & 24.5 & 0.0 & 0.0 & 0.0 & 35.4 & 0.0 & 0.0 & 0.0 & 7.7 \\
\hline $1.39 \mathrm{E}+08$ & 1.97E-01 & 0.0 & 0.0 & 30.8 & 0.0 & 0.0 & 27.1 & 0.0 & 0.0 & 7.1 & 0.0 & 0.0 & 0.0 \\
\hline $1.49 \mathrm{E}+08$ & $1.84 \mathrm{E}-01$ & 0.0 & 0.0 & 25.7 & 0.0 & 0.0 & 25.7 & 0.0 & 0.0 & 7.2 & 0.0 & 0.0 & 0.0 \\
\hline $1.72 \mathrm{E}+08$ & $1.59 \mathrm{E}-01$ & 0.0 & 0.0 & 24.3 & 0.0 & 0.0 & 22.9 & 0.0 & 0.0 & 6.3 & 0.0 & 0.0 & 0.0 \\
\hline $1.95 \mathrm{E}+08$ & 1.39E-01 & 0.0 & 0.0 & 27.0 & 0.0 & 0.0 & 18.2 & 2.9 & 0.0 & 3.7 & 2.5 & 0.0 & 0.0 \\
\hline $3.04 \mathrm{E}+08$ & $9.01 \mathrm{E}-02$ & 0.0 & 0.0 & 0.0 & 20.1 & 0.0 & 0.0 & 0.0 & 24.9 & 0.0 & 0.0 & 0.0 & 3.9 \\
\hline $4.77 \mathrm{E}+08$ & 5.73E-02 & 0.0 & 0.0 & 3.00 & 19.51 & 0.0 & 0.0 & 0.0 & 9.29 & 3.37 & 0.0 & 0.0 & 0.0 \\
\hline
\end{tabular}

Note: Left two columns give the Taylor and Rossby number. The table corresponds with Figure 17.6, right.

Figure 17.6 illustrates that EOF decomposition is a powerful tool to classify regime transitions. Such an EOFbased classification is not restricted to laboratory data but should work as well for data from the real atmosphere.

In this section, we have presented the application of multivariate statistical methods to velocity data, and we have highlighted the particular abilities of the CEOF analysis and the MSSA to detect interactions. In the next section, we will apply the CEOF analysis to a more complex experiment with broken azimuthal symmetry and time-dependent boundary conditions. Moreover, in addition to the velocity, surface temperature has been measured as well.

\subsubsection{Baroclinic Waves in Rotating Annulus with Barrier: CEOF Analysis}

Several experiments with the differentially heated rotating annulus have been performed with modifications to the standard geometry (flat bottom, constant gap width). These geometric modifications were introduced to better represent certain aspects of natural flows, e.g., to further understand the dynamics of zonal flow over topography [Weeks et al., 1997]. In ocean basins, the zonal flow is blocked by continents. Thus, several authors studied the case when a radial barrier is mounted in the annulus to understand better the western intensification of flows in 

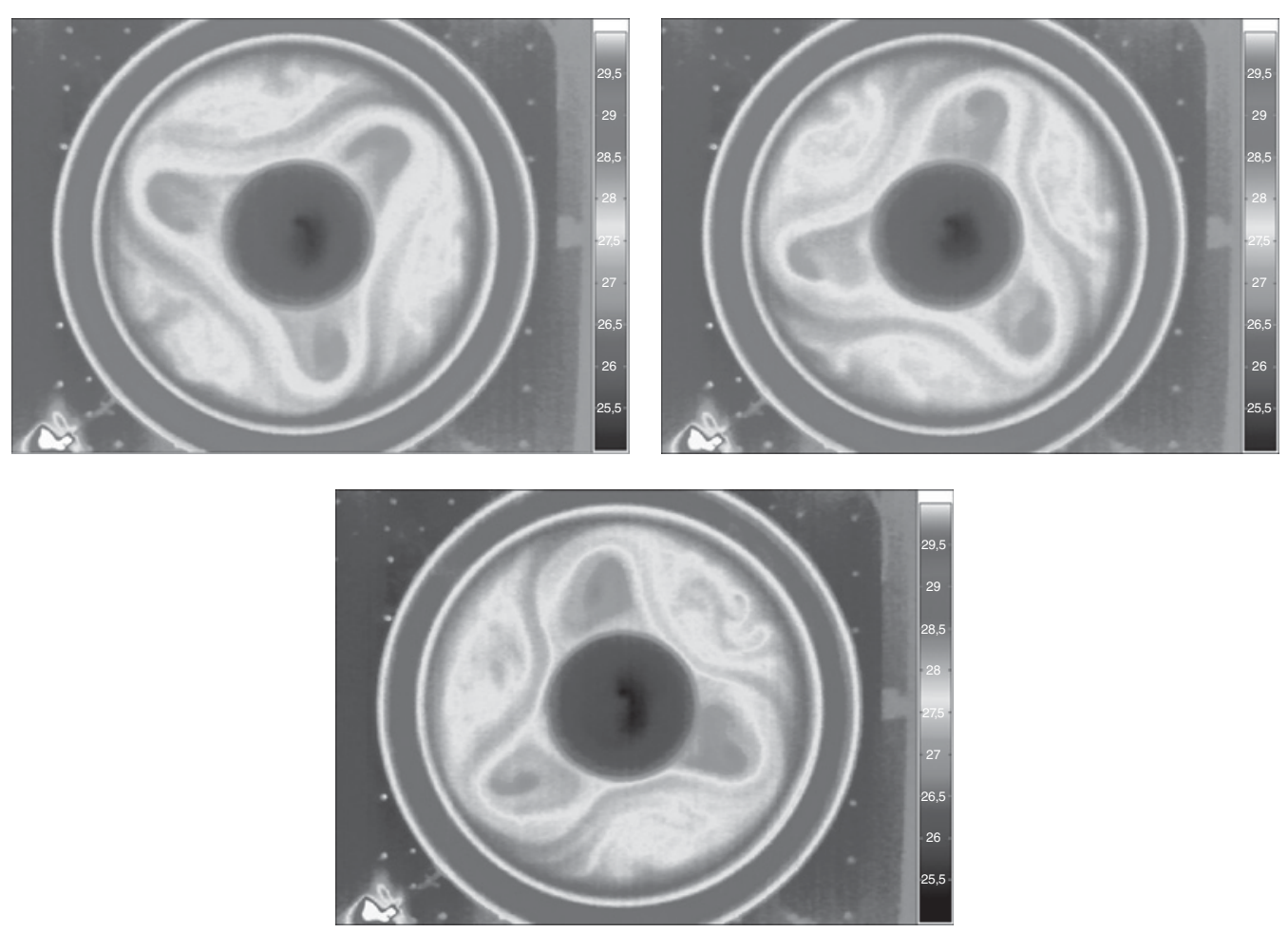

Figure 17.7. Sequence of surface temperature in the standard geometry at 69,73 , and 77 revolutions.

ocean basins [Bowden and Eden, 1968; Maxworthy and Browand, 1974; Rayer et al., 1998].

Not all oceanic flows are completely blocked in the zonal direction. The Antarctic Circumpolar Current (ACC), for example, is not blocked, but the flow has to weave through the Drake Passage between the southern tip of South America and the Antarctic continent. The Drake Passage partially blocks the zonal flow. It is therefore straightforward to perform experiments with partial barriers to understand better the intermediate case between a free and fully blocked annulus flow.

In the experiment discussed here, the azimuthal flow is blocked at the inner cylinder and at the bottom (see Figure 17.9). At the barrier, the gap width is reduced from 75 to $43 \mathrm{~mm}$ and the fluid depth is reduced from 135 to $95 \mathrm{~mm}$. More details can be found in the work of Harlander et al. [2012a].

Figure 17.7 shows a sequence of surface temperature images taken from an experiment in standard geometry with $\Omega=4.6 \mathrm{rpm}$ and $\Delta T=2.8 \mathrm{~K}$. We see the baroclinic wave after 69, 73, and 77 revolutions. Obviously, the wave is rather stable and rotates counterclockwise (that is, prograde) within a prograde mean flow. In contrast, in Figure 17.8, we see a comparable experiment ( $\Omega=4.8 \mathrm{rpm}$ and $\Delta T=4.0 \mathrm{~K}$ ) but then with the barrier mounted. The most obvious new feature is wave breaking at the barrier and wave recovery downstream of the barrier. That is, the baroclinic wave never saturates but is invariably in a transient state. We can say that the barrier leads to a mechanically induced baroclinic life cycle. Life cycles play an important role for midlatitude atmospheric and oceanic flows where they occur due to linear growth, nonlinear saturation, and dissipative decay of large-scale waves. The experiment with the barrier opens the possibility to study baroclinic life cycles in a controlled way.

The experiment we discuss shows a slow periodic variation of the radial temperature difference with an amplitude of $1 \mathrm{~K}$ and a period of $26 \mathrm{~min}$. The purpose of this variation of boundary conditions is to make sure that the wave breaking is due to the barrier and not due to regime transitions that occur for certain Taylor and Rossby numbers. We can exclude the latter from the fact that the flow looks very similar for maximum and minimum $\Delta T$. It is important to note that during the experiment we observed the surface velocity and surface temperature simultaneously. This allows for analyzing coupled temperature and velocity anomalies and the evaluation of the mean and turbulent surface heat flux.

The CEOF analysis already applied in Section 17.3.1 is eminently suited for our data [Pfeffer et al., 1990]. The CEOF analysis decomposes a propagating mode into a 

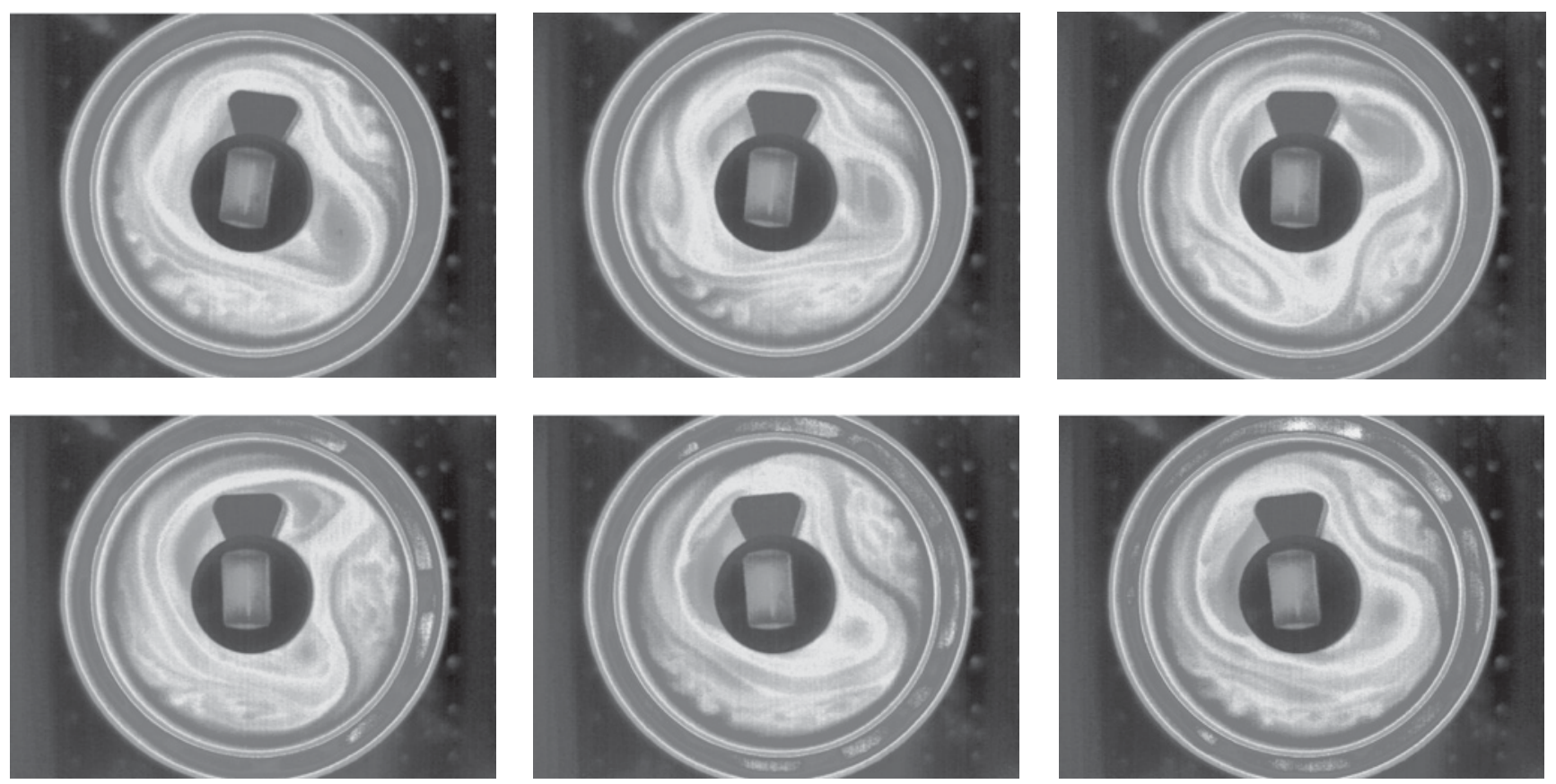

Figure 17.8. Sequence of surface temperature in the geometry with the barrier mounted. Top row from left to right: at 37,39 , and 43 rotations. Bottom row from left to right: at 45, 47, and 48 revolutions.

(a)

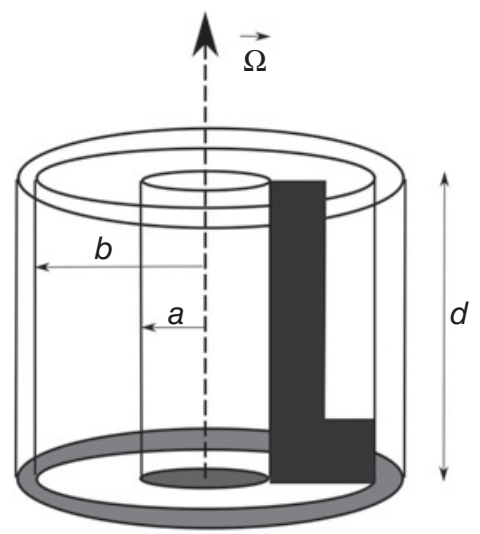

(b)

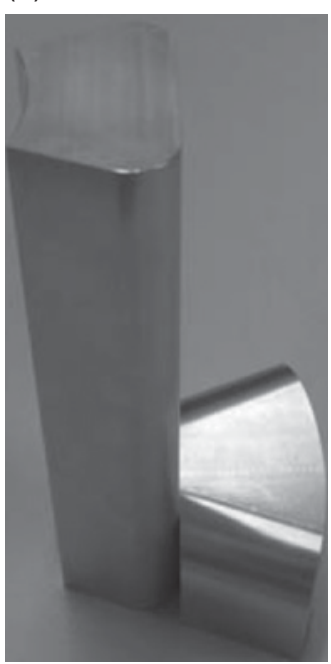

Figure 17.9. (a) Annulus with the barrier. (b) Photograph of the barrier.

single complex orthogonal function and not in two real functions. Therefore, amplitude and phase information is contained in a single CEOF. More specifically, we will apply a combined CEOF Analysis to find connected propagating anomalies of surface temperature and velocity [Harlander et al., 2012a]. Frequently, the dominant components of a high-dimensional process are captured with often surprisingly few "modes" and thus the CEOF analysis is an efficient technique for dimension reduction.

We computed CEOFs over the full annulus and over subregions. The first 10 eigenvalues denoting the variance comprised in the corresponding CEOFs of the annulus region upstream of the barrier are shown in Figure 17.10a. We find that the first two local CEOFs explain more than $50 \%$ of the total variance in the region upstream of the barrier. For the CEOFs of the full annulus we find a very similar spectrum with contributions of $28.8 \%$ and $19.4 \%$ for CEOF1 and CEOF2, respectively. $\mathrm{PC} 1$ and $\mathrm{PC} 2$, that is, the temporal behavior of CEOF1 and CEOF2, are shown in Figures 17.10b and 17.10c for the upstream region. Note that real and imaginary parts are in quadrature, indicating propagating anomalies. Obviously, PC2 comprises for a significant part the slow periodic variation of the radial temperature difference mentioned above. In contrast, PC1 shows a strong wave oscillation and only a weak modulation due to the slow variation of the temperature difference. We find about 9 wave oscillations per 100 rotations. The PCs of the full annulus look very similar to the one of the upstream part; however, the order of PC1 and PC2 is reversed. That is, for the full annulus the first PC mainly contains the low-frequency forcing.

We now focus on the dominant spatial structure of the anomalies. CEOF2 of the full annulus and CEOF1 of the local domain are shown in Figures 17.11a-d. The CEOFs shown correspond to the PCs that mainly contain the waves and not the low-frequency forcing. Real parts 
(a)

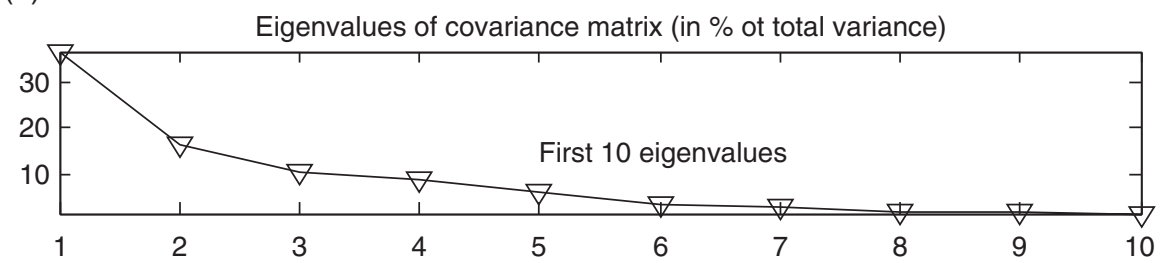

(b)

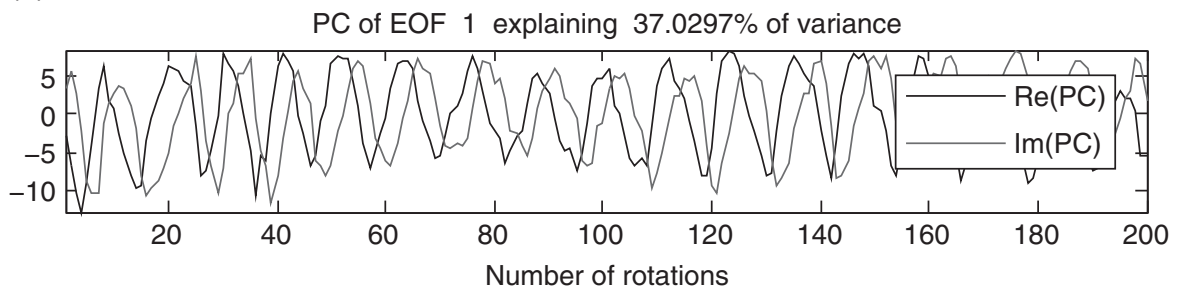

(c)

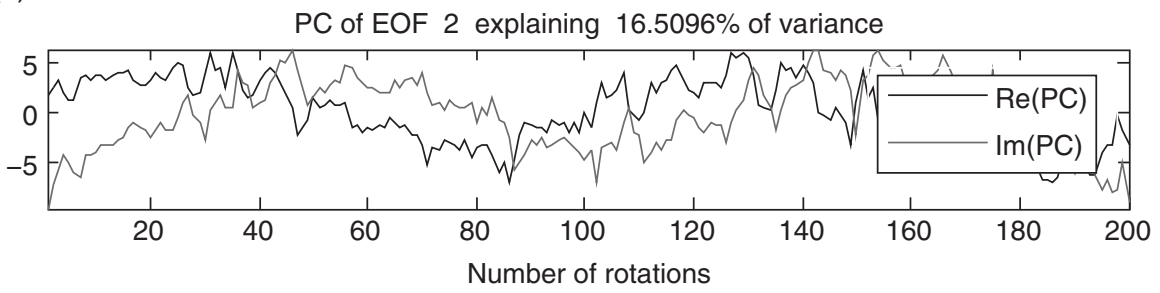

Figure 17.10. (a) Variance spectrum of a CEOF analysis. (b) Real and imaginary parts of PC1. (c) Real and imaginary parts of PC2. PC1 mainly captures the propagating wave whereas PC2 comprises a significant part of the low-frequency temperature forcing.

$(a, c)$ and imaginary parts $(b, d)$ of the CEOF show the propagating anomaly at two different phases with a phase shift of $\pi / 2$. The patterns have to be read in the following way: Figure 17.11a,c represent the flow at the beginning of a cycle, and Figures $17.11 \mathrm{~b}, \mathrm{~d}$ represent a quarter of a cycle later. The fields displayed in Figures 17.11a,c but multiplied by -1 show the flow at half of the cycle; Figure $17.11 \mathrm{~b}, \mathrm{~d}$ multiplied by -1 give the flow at three quarters of the cycle. A quarters of the cycle later the cycle is complete and the starting point is reached again (that is, Figures 17.11a,c).

CEOF1 computed for the full annulus (not shown) contains a mix of waves and the forcing signal and is more difficult to interpret than CEOF2. In the temperature field we find the modulation pattern as an increase/decrease of the temperature along the outer boundary. We also find propagating wave structures and vortices in the upstream region and a locally fixed but pulsating vortex in the downstream region. This vortex can be seen even clearer in a local EOF analysis of the downstream region and is likely directly connected to the barrier and is not generated by baroclinic instability. The strength of the vortex changes due to the oscillating meridional temperature contrast.
CEOF2 (Figures 17.11a,b) clearly reveals that the wave structure is prominent in the upstream and weak in the downstream region. The pattern of the local CEOF1 (Figures $17.11 \mathrm{c}, \mathrm{d}$ ) is rather easy to interpret. It is clearly dominated by a regular train of vortices slowly traveling prograde towards the barrier. Note that the local CEOFs have been computed independently from the full annulus just for the local domain. Still, Figures 17.11c,d resemble very closely the upstream part of the full annulus CEOFs (Figures 17.11a,b). From the local CEOFs we find that temperature anomalies are not circular but show a prominent bulge that is opposed to the direction of the mean flow. We also find that the temperature maxima and minima do not correspond with the centers of the vortices. With respect to these centers, the temperature anomalies are shifted toward the inner cylinder and slightly downstream. We see that due to the bulges, a significant positive heat flux can be observed (positive anomalies are transported inward, negative anomalies outwards). This flux reduces the radial temperature gradient.

From Figures 17.11c,d we see further that the strength of a vortex continuously increases until the anomaly reaches the barrier. We further note a small cyclone within the constriction of the annulus (Figure 17.11d). This 
(a)

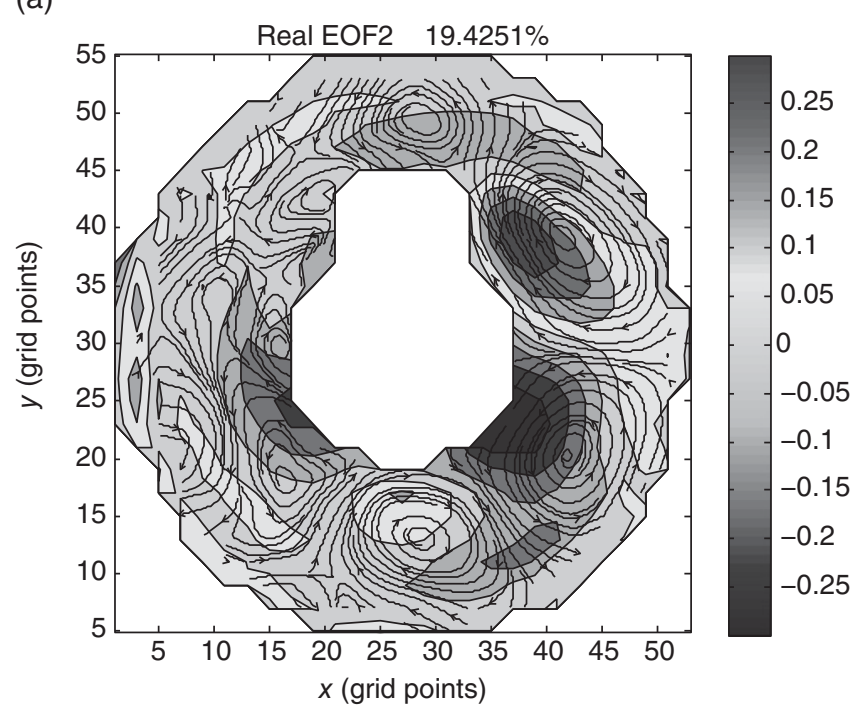

(c)

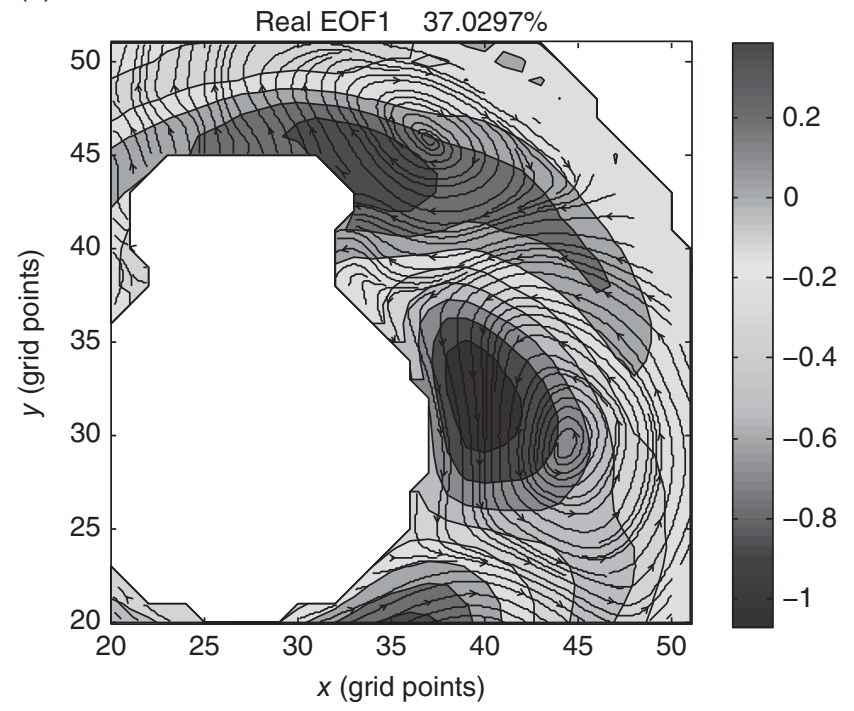

(b)

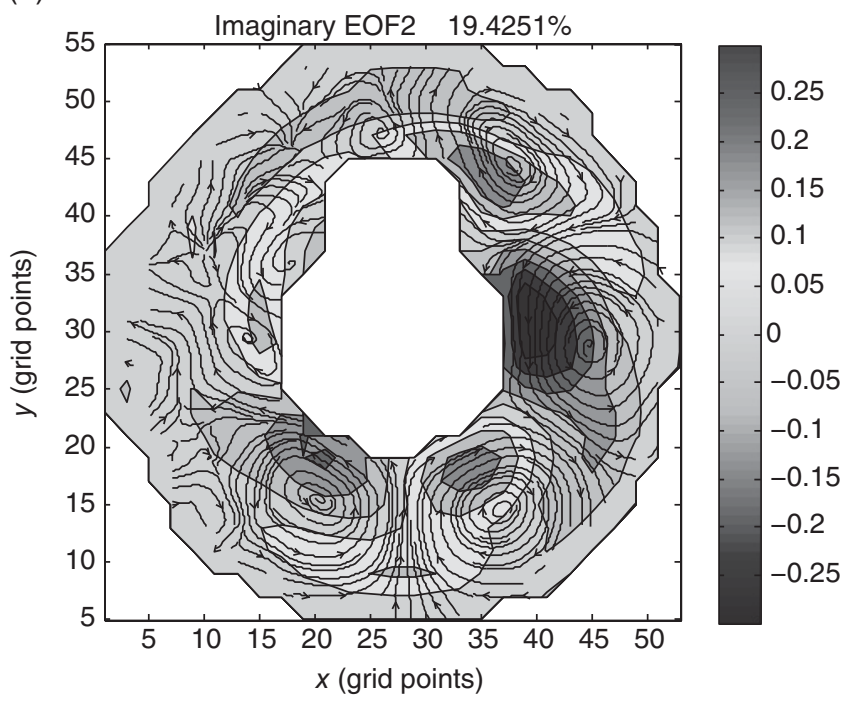

(d)

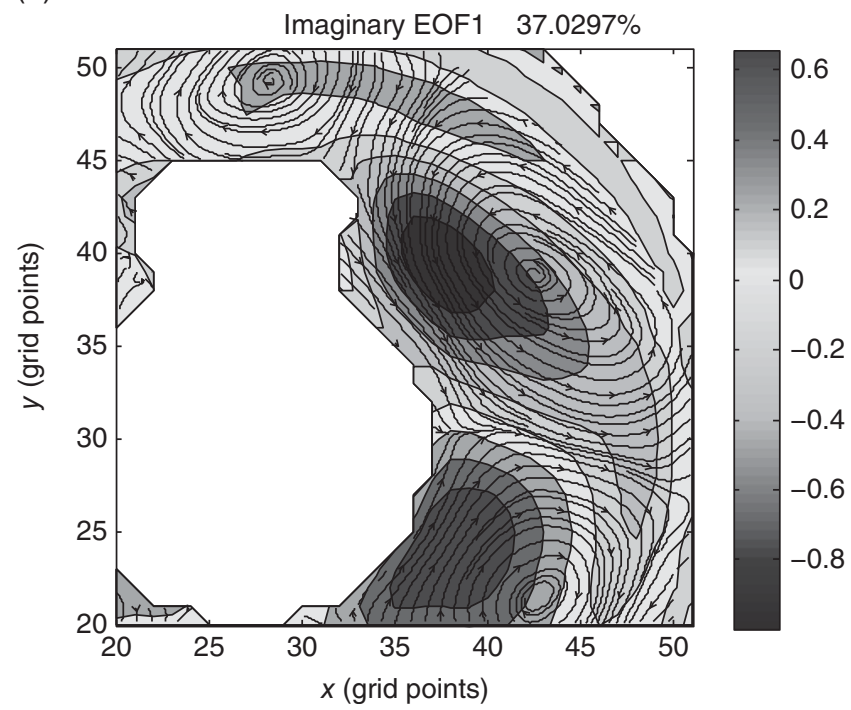

Figure 17.11. Real and imaginary parts of CEOFs: surface temperature and velocity: (a,b) CEOF2 for full annulus; (c,d) CEOF1 of upstream region. The CEOFs resolve coupled heat and velocity anomalies propagating anticlockwise toward the barrier. For color detail, please see color plate section.

demonstrates that vortices are important in the constriction even if this is not directly visible in Figure 17.8. However, the magnitude of the vorticity in the gap is weak compared to the vorticity in the region upstream of the barrier. Note finally that downstream of the barrier the anomalies become more turbulent and less coherent features can be observed (Figures 17.11a,b).

The present experiment of a differentially heated rotating annulus with a radial barrier that partially blocks the azimuthal component of the flow along the inner cylinder and the bottom enables us to present a rather general picture of the transient flow. This picture should hold for a certain range of Taylor and thermal Rossby numbers in the wave regime of the annulus. Roughly speaking, the annulus can be subdivided into the half upstream of the barrier, where waves amplify, and the half downstream of the barrier, where waves decay (see Figures 17.8 and 17.11). The dominant linear wave mode (in our case with azimuthal wave number 3 ) is unstable upstream but stable downstream of the barrier. In the upstream half, the azimuthal mean flow is moderate but with a significant positive radial eddy heat flux. In contrast, in the downstream half, we find an increased radial mean temperature gradient. The latter points to a weakened or 
even reversed radial eddy heat flux in the lee side of the barrier.

The experiment described has not been designed to resemble some particular large-scale flow that can be found in nature. However, it is tempting to point to similarities between experimental and real flows. The ACC is an annulus-like large-scale flow that is partially blocked at the Drake Passage, connecting the Pacific and the Atlantic oceans. For the ACC, eddy fluxes play a more central role in the dynamical and thermodynamical balances than in other oceans [Rintoul et al., 2001]. Of course, many processes important for the ACC dynamics are not captured by the experiment, e.g., wind stress, coastal as well as bottom topography, and the high-latitude $\beta$-effect [Harlander, 2005; Afanasyev et al., 2009, chapter 5 this issue]. Nevertheless, a meridional overturning circulation is present in the ACC and the experiment and baroclinic instability plays an important role for the ACC dynamics due to the meridional transport of heat toward the pole.

\subsubsection{Principal Oscillation Patterns and Singular Vectors}

In many cases, EOFs can be interpreted as the patterns of natural oscillations, i.e., as the eigenmodes of the system under consideration. From a mathematical point of view such an interpretation is not justified. However, empirically estimating the eigenmodes and then comparing them with the EOFs can reveal the connection between modes and patterns of variability [Harlander et al., 2009b]. Empirically estimated normal modes are called principal oscillation patterns (POPs) in the meteorological literature [Hasselmann, 1988]. In the following we will compare EOFs and POPs computed from our annulus data. In a subsequent step we will then estimate optimal growing initial perturbations, called singular vectors from the POPs. Theoretically, SVs converge to POPs in the limit $t \rightarrow \infty$. As we will see, this holds for the empirically estimated SVs as well. SV growth might explain the increased irregularity that occurs for annulus flows at Taylor numbers larger that $10^{8}$. We will briefly discuss this by employing laboratory data and model results.

17.3.3.1. Principal Oscillations Patterns. Generally speaking, the POP method is promising when the dynamical process under consideration is linear to first approximation. This sounds very restrictive in particular when geophysical applications are the target. However, the method has successfully been applied to a wide class of geophysical data [von Storch and Zwiers, 1999].

The linear system considered reads

$$
\mathbf{x}(t+\Delta)=\mathbf{G}(\Delta) \mathbf{x}(t),
$$

where $\mathbf{x}(t+\Delta)$ is the state vector at time $t+\Delta$ and $\mathbf{G}(\Delta)$ the propagator matrix that maps the state at time $t$ to time $t+$ $\Delta . \mathbf{G}(\Delta)$ bears the argument $\Delta$ since it can be estimated by

$$
\mathbf{G}(\Delta)=\mathbf{C}(\Delta) \mathbf{C}(0)^{-1}
$$

where

$$
\begin{aligned}
\mathbf{C}(\Delta) & =\left(\mathbf{F}^{1 \Delta}\right)^{T} \mathbf{F}^{0 \Delta}, \\
\mathbf{C}(0) & =\left(\mathbf{F}^{0 \Delta}\right)^{T} \mathbf{F}^{0 \Delta}
\end{aligned}
$$

are the covariance matrices with lag $\Delta$ and lag zero. The eigenvectors of $\mathbf{G}(\Delta)$ are the POPs, whereas the eigenvectors of $\mathbf{C}(0)$ are the EOFs. Usually, the POPs are sorted (in decreasing order) with respect to the e-folding times $\tau_{i}=-1 / \ln \left(\left|\lambda_{i}\right|\right)$, where the $\lambda_{i}$ are the eigenvalues of $\mathbf{G}(\Delta)$. The period of the POP is given by $T=2 \pi / \arg \lambda$.

We used surface temperature data to compute EOFs and POPs. The data have been recorded by an infrared camera that has a noncooled microbolometer detector with a spectral range of $7.5-14.0 \mu \mathrm{m}$ and a temperature resolution smaller than $0.08 \mathrm{~K}$ with an accuracy of $\pm 1.5 \mathrm{~K}$ at $30^{\circ} \mathrm{C}$. The spatial resolution of the infrared sensor is $640 \times 480$ pixels. To reduce the size of the covariance matrices we smoothed the data by using a running average over areas of $2 \times 2$ pixels. In Figure 17.12a we display the EOF1 that explains $27 \%$ of the total variance. Further, in Figure 17.12b, the real part of the least damped POP1 with a damping time of $1827.6 \mathrm{~s}$ and a period of $62.5 \mathrm{~s}$ for an experiment with $\Omega=6 \mathrm{rpm}$ and $\Delta T=8 \mathrm{~K}$ is shown. It should be noted that EOF2 is a phase shifted version of EOF1 with nearly the same explained variance. Obviously, EOF1 and POP1 agree very well. Taking EOF1 and EOF2, as well as the real and imaginary parts of POP1 together, both patterns propagate with the same phase speed. This suggests that for the data considered the EOFs represent the eigenmodes of the system.

Let us next use the POPs to estimate the dominant patterns of nonmodal instability. This procedure needs an appropriate filtering and the EOF analysis is a suitable method for this purpose.

17.3.3.2. Empirical Singular Vectors. Instability is related to exponentially growing eigenmodes and thus to POPs with $\left|\lambda_{i}\right|>1$. Interestingly, when finite time intervals are considered, growth rates of certain initial perturbations can exceed the growth rates of the most unstable modes. Moreover, even when all modes are damped ( $\left.\left|\lambda_{i}\right|<1\right)$, such particular initial perturbations can grow dramatically during finite time intervals. The perturbations with the largest growth rates are called singular vectors or optimal perturbations. They play an important role not only in atmospheric ensemble predictions [Kalnay, 2002] but also for the theory of instability and turbulence [Trefethen et al., 1993; DelSole, 2007]. 
(a)

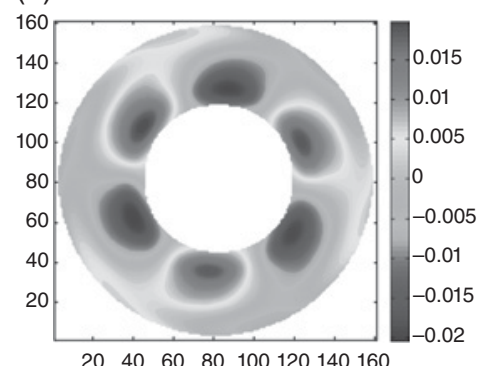

(d) (b)

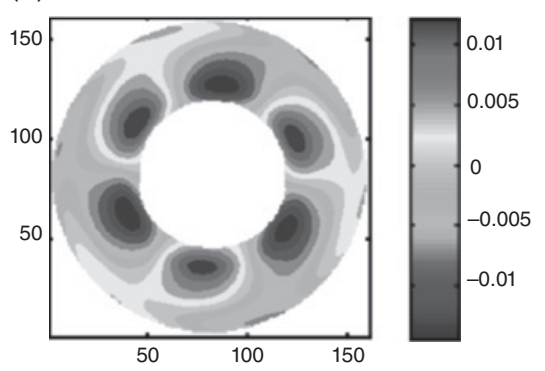

(c)

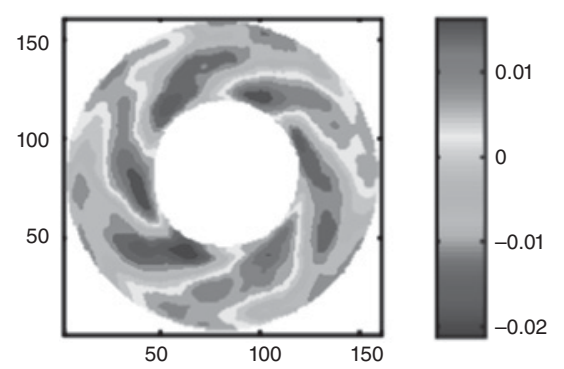

(d)

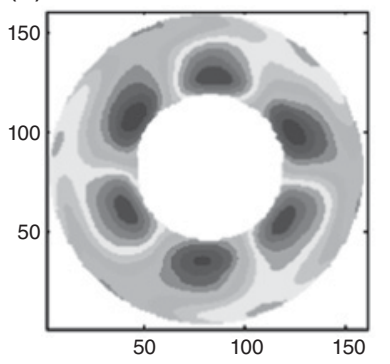

(e)

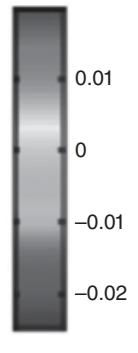

(e)

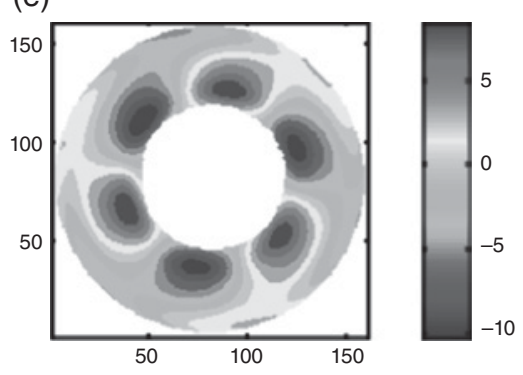

Figure 17.12. Experiment with parameters $\Omega=6 \mathrm{rpm}, \Delta T=8 \mathrm{~K}$. (a) EOF1, 27\% explained variance. (b) POP1, damping time $1827.6 \mathrm{~s}$, period $62.5 \mathrm{~s}$. (c) First singular vector at $t=0, t_{\mathrm{opt}}=20 \mathrm{~s}, \sigma=1.182$. (d) First singular vector at $t=t_{\mathrm{opt}}$. (e) First singular vector at $t=2500 \mathrm{~s}$.

The starting point for the SV analysis is the linear dynamical system

$$
\frac{d \mathbf{x}}{d t}=\mathbf{B} \mathbf{x}
$$

with the system matrix B. In atmospheric ensemble prediction linearization is generally done around a nonlinear solution and then $\mathbf{B}$ is time dependent. In contrast, in turbulence research linearization is done around a mean state. In that case $\mathbf{B}$ is time independent [DelSole, 2007]. We consider the mathematically simpler latter case.

The matrices $\mathbf{G}$ in (17.7) and $\mathbf{B}$ in (17.10) are connected via the equation

$$
\mathbf{G}(\Delta)=\exp (\mathbf{B} \Delta)=\sum_{k=0}^{\infty} \frac{1}{k !}(\mathbf{B} \Delta)^{k}
$$

or

$$
\mathbf{B}=\frac{1}{\Delta} \ln [\mathbf{G}(\Delta)]=\frac{1}{\Delta} \sum_{k=1}^{\infty} \frac{(-1)^{k+1}}{k}[\mathbf{G}(\Delta)-\mathbf{E}]^{k}
$$

where $\mathbf{E}$ is the identity matrix. It is instructive to note that by discretizing the first term in (17.10) by $[\mathbf{x}(t+\Delta)-$ $\mathbf{x}(t)] / \Delta$ and using (17.6), we find

$$
\mathbf{B}=\frac{1}{\Delta}[\mathbf{G}(\Delta)-\mathbf{E}] .
$$

This corresponds to (17.12) when just the first term is kept. The simplification gives still good results for low-dimensional systems [Harlander et al., 2009a] but it fails in general.

Having estimated the system matrix B from (17.12), we can compute the propagator $\mathbf{G}$ for any time interval $\Delta_{0}$ by

$$
\mathbf{G}\left(\Delta_{0}\right)=\exp \left(\mathbf{B} \Delta_{0}\right)
$$

When the $L_{2}$-norm is used to measures the growth of a perturbation in the time interval $0 \leq t \leq \Delta_{0}$, the SVs with optimazation time $\Delta_{0}$ are given by the eigenvectors of the matrix $\mathbf{G}^{T}\left(\Delta_{0}\right) \mathbf{G}\left(\Delta_{0}\right)$. The eigenvalues of the matrix define the square of the growth rates.

Figure $17.12 \mathrm{c}$ shows the first singular vector at $t=0$ estimated from surface temperature data of an experiment with $\Omega=6 \mathrm{rpm}$ and $\Delta T=8 \mathrm{~K}$. The data sampling rate was $\Delta=5 \mathrm{~s}$, and the optimization time was set to be $\Delta_{0}=$ $20 \mathrm{~s}$. For the time interval $0 \leq t \leq \Delta_{0}$ we obtain a growth rate of $\sigma=1.182$. To reduce the noise in the data, we used the EOF filtering technique. Just 33 EOFs explain a total variance very close to $90 \%$. Thus we used $j=33$ in (17.5) to reconstruct the data from the EOFs. It should be noted that the growth rate increases by increasing the number of EOFs used for the reconstruction. It appears that the more EOFs that are available to support the SVs, the larger is the maximum growth rate. However, reconstructing the data by a large number of EOFs increases the noise, and at a certain number, the SVs seem to be dominated by noise and lose their physical meaning. 
Figure 17.12d shows SV1 at the optimization time $t=\Delta_{0}$ computed by $\mathbf{x}\left(\Delta_{0}\right)=\mathbf{G}\left(\Delta_{0}\right) \mathbf{x}(0)$, where $\mathbf{x}(0)$ is the $\mathrm{SV}$ at $t=0$ shown in Figure 17.12c. Obviously, the tilted troughs and ridges have turned up as expected [Will et al., 2006]. The structure does not change much for longer times. Figure 17.12e shows the SV after $t=125 \Delta_{0}=$ 2500 s computed by $\mathbf{x}\left(N \Delta_{0}\right)=\mathbf{G}^{N}\left(\Delta_{0}\right) \mathbf{x}(0)$ with $N=$ 125. As expected, this pattern agrees very well with POP1 shown in Figure 17.12b. For $t \rightarrow \infty$ the SVs converge to the corresponding normal modes [Kalnay, 2002].

Seelig et al. [2012] discussed SVs of the simple Lorenz annulus model [Lorenz, 1984] in the context of transitions to irregular flow and compared some numerically deduced SVs with data-based ones. The numerical model enabled the construction of a regime diagram in terms of singular vector growth rates, where the abscissa was the Taylor number and the ordinate the thermal Rossby number. Strikingly, the diagram based on singular vector growth strongly resembles the traditional bifurcation diagram for annulus flows [Hide and Mason, 1970; Lorenz, 1984]. The largest growth rates could be found in the irregular flow regime of the Lorenz model.

The findings from the simple numerical model suggest that the gradual increase of irregularity in the rather broad transition region to quasi-geostrophic turbulence might partly be addressed to singular vector growth. For laboratory experiments as well as for natural flows there is always a certain background noise level. Irregularities in the transition region might be seen as extreme events that arise from random excitation of singular vectors with unusual large growth rates [DelSole, 2007]. This process, together with nonlinear wave-wave interaction, could explain the gradual broadening of the spectrum when the rotating annulus flow transits to geostrophic turbulence [Pfeffer et al., 1997]. Whether these ideas, derived from the loworder Lorenz model, can be transferred to real annulus flows is not clear yet. More data sets have to be analyzed by the techniques described above to address this question. However, it can be expected that the growth rates increase for irregular flows since more EOFs have to be considered to cover, say, $90 \%$ of the total variance for irregular flows.

\subsubsection{Helmholtz-Hodge Decomposition of Annulus Flows}

According to the Helmholtz-Hodge decomposition theorem, any suitably smooth vector field can be decomposed into the sum of a divergence-free field and a curl-free field [Foias et al., 2008]. These two fields can be used to discriminate different wave types occurring in the annulus. Baroclinic waves and Rossby waves are divergence free, whereas inertia-gravity waves comprise a significant part of horizontal divergence. Presently, the process of spontaneous gravity wave emission is a major issue in atmospheric research. The differentially heated rotating annulus is a lab experiment suitable to systematically study spontaneous gravity wave emission in analogy to the atmosphere [Williams et al., 2008]. To detect inertialgravity waves in the experimental data, it is favorable to not use the full flow field but instead make use of the decomposition and analyze just the curl-free part of the flow.

The primary difficulties with computing the decomposition of the measured horizontal velocity at a given level of the cylindrical tank is that the PIV data do not line up on a nice grid, and the data may contain noise. To handle these two issues, we use a mesh-free reconstruction method based on radial basis functions (RBFs). The method employs matrix-valued kernels [Narcowich and Ward, 1994] and mimics the Helmholtz-Hodge decomposition of a $2 \mathrm{D}$ velocity field. It is similar to the method described by Fuselier and Wright [2009] for the surface of the sphere but is instead adapted for a 2D annular domain, for which dealing with boundaries becomes important. The method also provides a means of filtering the noise in the measured velocity fields and can be used to reconstruct the full 3D field in the rotating annulus. The key ingredients to the mesh-free reconstruction and decomposition technique are divergence-free and curl-free matrixvalued kernels. In this study, we construct these kernels from the scalar-valued Matérn radial kernels, which are popular for spatial statistics [Stein, 1999] and are given by

$$
\begin{aligned}
\phi_{\nu}(r)=\frac{1}{2^{v+1} \Gamma(v+1)}(\alpha r)^{v} K_{v}(\alpha r), & \\
r & \geq 0, \quad v>\frac{5}{2}, \alpha>0,
\end{aligned}
$$

where $K_{v}$ is the modified Bessel function of the second kind of order $v$. Increasing $v$ in (17.15) increases the smoothness of the kernel, while increasing $\alpha$ increases its peakedness. Letting $\mathbf{x}=(x, y)$ and $\mathbf{x}_{j}=\left(x_{j}, y_{j}\right)$, the respective divergence-free and curl-free matrix-valued kernels are then defined as [Narcowich and Ward, 1994]

$$
\begin{aligned}
\Phi_{v}^{\mathrm{div}}\left(\mathbf{x}, \mathbf{x}_{j}\right) & =\left(-\nabla^{2} \mathbf{I}+\nabla \nabla^{T}\right) \phi_{\nu}\left(\left\|\mathbf{x}-\mathbf{x}_{j}\right\|_{2}\right), \\
\Phi_{v}^{\mathrm{curl}}\left(\mathbf{x}, \mathbf{x}_{j}\right) & =-\nabla \nabla^{T} \phi_{v}\left(\left\|\mathbf{x}-\mathbf{x}_{j}\right\|_{2}\right),
\end{aligned}
$$

where $\mathbf{I}$ is the $2 \times 2$ identity matrix and $\nabla \nabla^{T}$ is the Hessian matrix. By construction, the columns of $\Phi_{v}^{\mathrm{div}}$ are divergence free, while the columns of $\Phi_{v}^{\text {curl }}$ are curl free.

Before discussing the exact details on the reconstruction and decomposition method, we note that since the present application involves boundaries, it is necessary to supplement the given data with boundary conditions to make the decomposition of 2D velocity field unique [Foias et al., 2008]. We assume that both the divergence-free and curl-free parts of the field are parallel to the boundaries. 
We enforce this condition on the reconstructed field at discrete locations on the boundary of the annulus.

Let $\mathbf{v}_{j}=\left(u_{j}, v_{j}\right), j=1, \ldots, N$, denote the normalized PIV measurements of the horizontal velocity field at any horizontal level $\ell$ of the cylindrical tank and let $\mathbf{x}_{j}=\left(x_{j}, y_{j}\right)$ denote the corresponding normalized locations of the measured field. Here we have normalized so that the outer radius of the tank is unity. Since the PIV data do not include measurements on the boundary, we must define these points. We choose the boundary points to be equally spaced on the inner and outer circles of the annulus with a density that is comparable to that of the interior points. We denote these boundary points by $\xi_{k}$, $k=1, \ldots, M$, and we let $\mathbf{n}_{k}$ denote the corresponding unit outward normal vector at $\xi_{k}$. The matrix-valued kernel approximation of the field then takes the form

$$
\begin{aligned}
\widetilde{\mathbf{v}}\left(\mathbf{x} ; v_{n}, v_{f}\right)= & \underbrace{\sum_{j=1}^{N} \Phi_{v_{n}}^{\operatorname{div}}\left(\mathbf{x}, \mathbf{x}_{j}\right) \mathbf{a}_{j}+\sum_{k=1}^{M}\left(\Phi_{v_{f}}^{\operatorname{div}}\left(\mathbf{x}, \boldsymbol{\xi}_{k}\right) \mathbf{n}_{k}\right) d_{j}}_{\widetilde{\mathbf{v}}^{\mathrm{div}}\left(\mathbf{x} ; v_{n}, v_{f}\right)} \\
& +\underbrace{\sum_{j=1}^{N} \Phi_{v_{n}}^{\mathrm{curl}}\left(\mathbf{x}, \mathbf{x}_{j}\right) \mathbf{a}_{j}+\sum_{k=1}^{M}\left(\Phi_{v_{f}}^{\mathrm{curl}}\left(\mathbf{x}, \boldsymbol{\xi}_{k}\right) \mathbf{n}_{k}\right) c_{j}}_{\widetilde{\mathbf{v}}^{\operatorname{curl}}\left(\mathbf{x} ; v_{n}, v_{f}\right)},
\end{aligned}
$$

where $\mathbf{a}_{j}=\left[\begin{array}{ll}a_{j} & b_{j}\end{array}\right]^{T}, c_{k}$, and $d_{k}$ are determined by the following constraints:

$$
\begin{aligned}
\widetilde{\mathbf{v}}\left(\mathbf{x}_{i} ; v_{n}, v_{f}\right)=\mathbf{v}_{i}, & i=1, \ldots, N, \\
\widetilde{\mathbf{v}}^{\operatorname{div}}\left(\xi_{i} ; v_{f}, v_{f}\right) \cdot \mathbf{n}_{i}=0, & i=1, \ldots, M, \\
\widetilde{\mathbf{v}}^{\operatorname{curl}}\left(\boldsymbol{\xi}_{i} ; v_{f}, v_{f}\right) \cdot \mathbf{n}_{i}=0, & i=1, \ldots, M .
\end{aligned}
$$

These constraints can be arranged into a $(2 N+2 M) \times$ $(2 N+2 M)$ symmetric linear system of equations for determining the unknown coefficients.

We have introduced two smoothness parameters $v_{n}$ and $v_{f}$ in (17.18) to provide a mechanism for filtering the reconstructed field. The method we use for filtering is adapted from a technique first proposed by Beatson and Bui [2007] for scalar-valued RBF approximations. It involves fitting the noisy data with one smoothness parameter $v_{n}$ and then evaluating the resulting approximation with a larger smoothness parameter $v_{f}$. This means the data is fit with one kernel but evaluated with a smoother yet similar kernel. As discussed by Beatson and Bui [2007], this kernel replacement technique corresponds to applying a low-pass filter to the approximations. Since the measurements are noisy and the boundary conditions are not, we only use $v_{n}$ in (17.19) when fitting the measurements. All evaluations of $\widetilde{\mathbf{v}}$ are done with $v_{n}=v_{f}$ to filter out the noise. The resulting filtered approximation then satisfies the boundary conditions. Presently, there is no theory for selecting $v_{n}$ and $v_{f}$ in an "optimal" manner. Instead, the choice is somewhat by trial and error. In the experiments that follow, we found that $v_{n}=3.5$ and $v_{f}=5.5$ gave good results for several different flow parameter regimes and vertical measurement levels. Half-integer choices for the smoothness parameter also lead to significant simplifications in computing (17.15) [Fasshauer, 2007].

Because of the properties of $\Phi_{v}^{\text {div }}$ and $\Phi_{\nu}^{\text {curl }}$, the expansions $\widetilde{\mathbf{v}}^{\text {div }}$ and $\widetilde{\mathbf{v}}^{\text {curl }}$ in (17.18) are divergence and curl free, respectively. Thus, the $\widetilde{\mathbf{v}}$ mimics the Helmholtz-Hodge decomposition theorem. Furthermore, an approximation to the divergence-free or curl-free parts of the field can be obtained from these respective expansions.

In Figure 17.13, we show the reconstruction and decomposition of the velocity field for two sets of parameters measured with PIV close to the surface at $z=120 \mathrm{~mm}$. For these data we set the shape parameter to $\alpha=20.91$, which corresponds to the inverse of the minimum of the pairwise distances between the normalized sample locations.

Figures 17.13a,c show contour plots of the streamfunction for the divergence-free part $\widetilde{\mathbf{v}}^{\text {div }}$ of the $120 \mathrm{~mm}$ fields, while Figures 17.13b,d show contours of the velocity potentials for the curl-free part of the fields $\widetilde{\mathbf{v}}^{\text {curl }}$. As can be seen, the main pattern of the flow is quasigeostrophic dynamics which is divergence free. The curlfree patterns shown in $17.13 \mathrm{~b}, \mathrm{~d}$ can be interpreted as a deviation from pure quasi-geostrophic flow. We see that these deviations are strongest at the inner and outer boundaries of the annulus. There prominent axial flows can be expected due to the heating and cooling of the boundaries. The axial gradients of this flow component induce a horizontal divergence. While the divergence-free part shown in Figures 17.13a,c is rather robust, the curlfree part is more delicate and already small effects can perturb the symmetry of the patterns. Still, the curl-free part of both experiments is rather smooth and no small-scale wavelike features can be seen. The reason for this might be that the spatial resolution of the PIV observations is not high enough to resolve the transient, nongeostrophically balanced part of the flow.

We conclude this section by noting that we can also use (17.18) to compute the divergence of the reconstructed velocity field at any location in the 2D slice of the cylindrical tank. These approximations can be combined with the incompressibility assumption of the full 3D fluid in the rotating annulus to reconstruct the full velocity field of the fluid (see Harlander et al. [2012b] and the extended abstract on http://ltces.dem.ist.utl.pt/lxlaser/ 1xlaser2012/upload/92_paper_ecvgbw.pdf for details). 
(a)

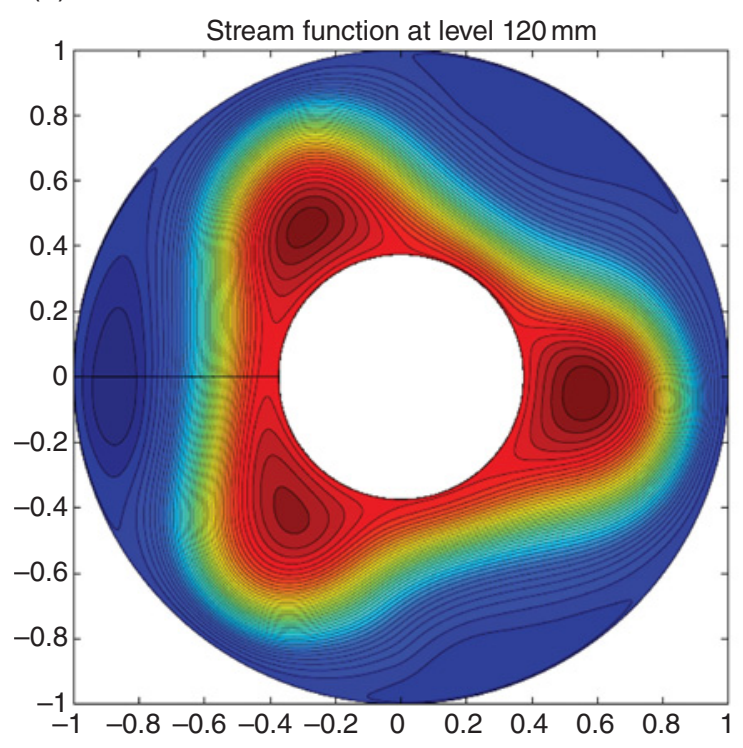

(c)

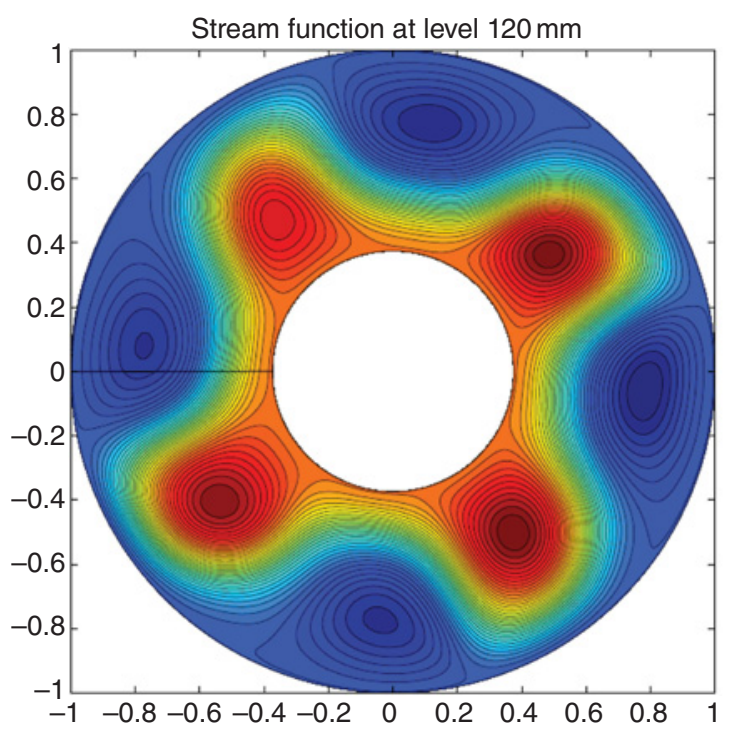

(b)
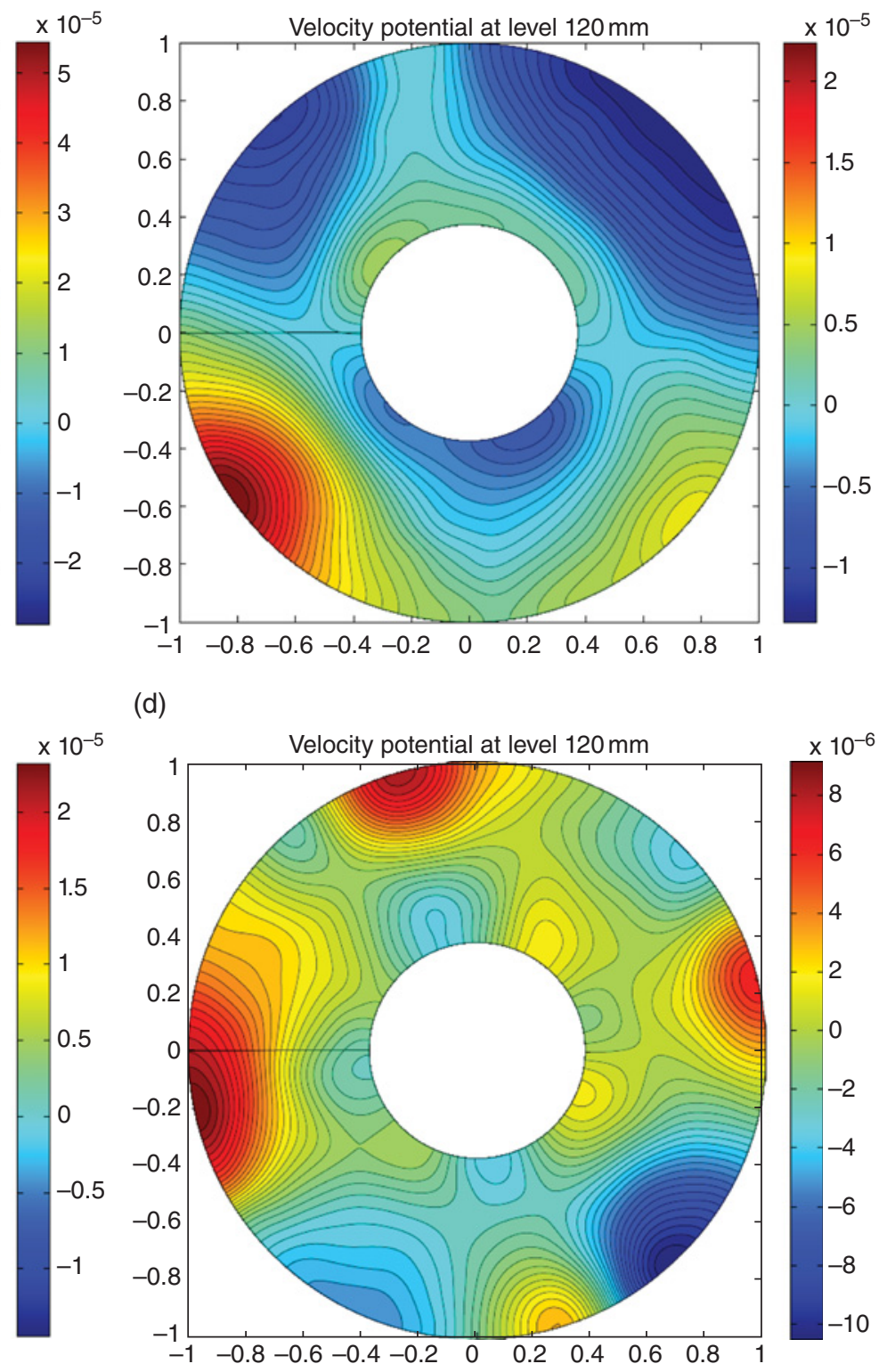

(d)

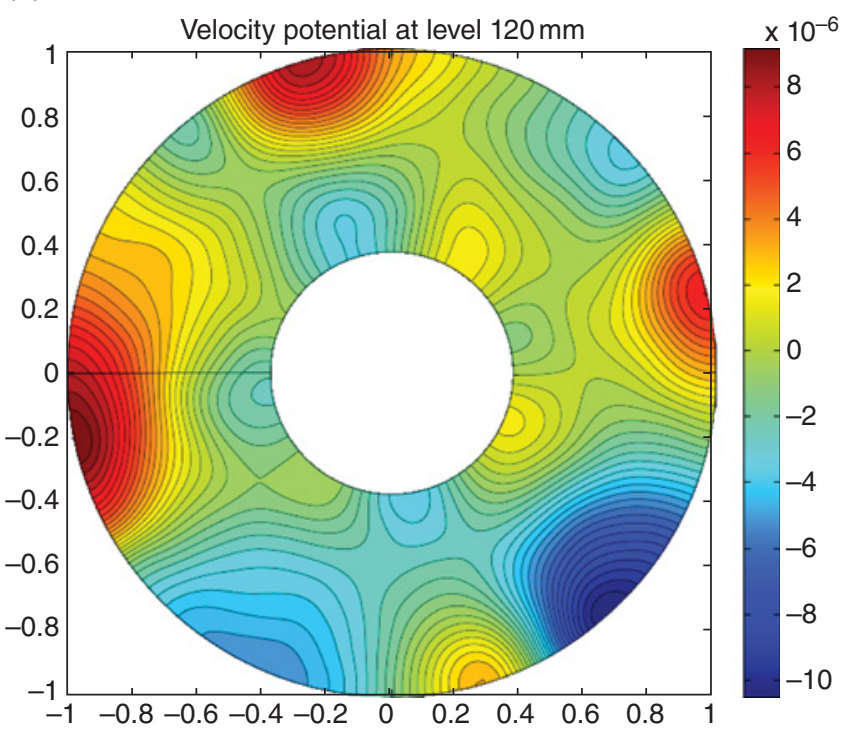

Figure 17.13. Helmholtz-Hodge decomposition for two sets of parameters $(\Omega, \Delta T, \mathrm{Ta}, \operatorname{Ro}, m)=\left(5.02 \mathrm{rpm}, 6.7 \mathrm{~K}, 1.93 \times 10^{7}\right.$, $1.189,3)$ for (a) and (b) and $(\Omega, \Delta T, T a, R o, m)=\left(15.00 \mathrm{rpm}, 8.0 \mathrm{~K}, 1.72 \times 10^{8}, 1.150,4\right)$ for (c) and (d): (a) stream function $m=3$, (b) velocity potential $m=3$, (c) stream function $m=4$, (d) velocity potential $m=4$.

\subsection{CONCLUSION}

This chapter summarized recent experimental work on the differentially heated rotating annulus. Some experimental techniques used are unique and the work on singular vectors and the Helmholtz-Hodge decomposition is novel. All the statistical and mathematical techniques discussed are versatile and can be applied to any kind of multivariate data, no matter whether they result from numerical models or field or laboratory observations.
Here we have applied the techniques solely on data from the heated rotating annulus that is operated in the lab of the Brandenburg University of Technology in Cottbus as part of the Multiple Scales in Fluid Mechanics and Meteorology initiative.

With the focus on geophysical fluid dynamics, the differentially heated and rotating annulus is an ideal test bed that allows for a rigorous and replicable testing of theories and computational tools. The atmosphere and oceans as well as many astrophysical fluids are stratified 
and rotate. The complex wave interactions and instabilities that shape their natural systems all have their equivalent in the annulus experiment.

Our understanding of the annulus flow is by far not complete and the work discussed in the present chapter can be seen as a starting point for further studies on the many facets of the experiment.

In Section 17.3.1 we studied transient features of baroclinic waves by using PIV and LDV measurements. The data sets involved could be expanded by simultaneous PIV and LDV observations. LDV is well suited to resolve fast local features like gravity waves excited close to the inner cylinder. At the same time, PIV can capture the state of the large-scale baroclinic wave that is particularly favorable for gravity wave excitation.

In Section 17.3.2 we discussed simultaneous surface temperature and PIV measurements. From these data the surface eddy heat flux can be derived [Harlander et al., 2012a]. A future systematic study of the surface eddy heat flux for different flow regimes and annulus geometries would help to understand better the transient eddies and their feedback on the mean flow [Wilson and Williams, 2006].

In Section 17.3.3 patterns with large growth rates, so-called singular vectors, have been estimated from data [Penland and Sardeshmukh, 1995]. To our knowledge, no method is available yet that can do this for systems with time-dependent system matrices. In that case, linearization can no longer be done about a time mean state. Instead, linearization about a full nonlinear realization of the flow is necessary. Due to its reproducibility, the annulus experiment is well suited to provide data to test future statistical methods that can handle problems with time-dependent system matrices.

Finally, as already mentioned at the end of Section 17.3.4, from simultaneous observed surface temperature and $2 \mathrm{D}$ flow measurements, the 3D flow can be reconstructed by using radial basis functions [Harlander et al., 2012b]. To further increase the reliability of such a novel reconstruction, the technique should be tested against stereo PIV observations that give 3D velocity fields on 2D laser slices. Combining such 3D flow observations with numerical simulations is a promising strategy to detect gravity waves and the excitation mechanisms in the stratified annulus [Williams et al., 2008; Scolan et al., 2013].

Acknowledgments. The work by Harlander, von Larcher, Alexandrov, and Egbers was partly funded by the German Science Foundation (DFG) under the DFG priority program MetStröm (SPP 1276/1-3) (grant EG 100/13-1-3). They thank Ulrich Achatz, Martin Baumann, Andreas Dörnbrack, Jochen Fröhlich, Vincent Heuveline, Stefan Hickel, Illia Horenko, Rupert Klein,
Oswald Knoth, and Eric Severac from the MetStröm rotating annulus group for many helpful discussions and Yongtai Wang for help regarding measurements and data handling. In particular the authors thank Emilia Crespo del Arco and Mani Mathur for helpful comments that improved the chapter. The work of Wright was funded in part by the U.S. National Science Foundation (NSF) under grant DMS-0934581. Wright also wishes to thank Edward Fuselier of High Point University for discussing various ideas related to Helmholtz-Hodge decompositions on bounded domains.

\section{REFERENCES}

Afanasyev, Y. D., P. B. Rhines, and E. G. Lindahl(2009), Velocity and potential vorticity fields measured by altimetric imaging velocimetry in the rotating fluid waves, Exp. Fluids, 47, 913-926.

Badger, J., and B. J. Hoskins (2001), Simple initial value problems and mechanisms for baroclinic growth, J. Atmos. Sci., $58,38-49$.

Barcilon, A., and P. G. Drazin (1984), A weakly nonlinear theory of amplitude vacillation and baroclinic waves, J. Atmos. Sci., 22, 3314-3330.

Beatson, R. K., and H. Q. Bui (2007), Mollification formulas and implicit smoothing, Adv. Computat. Math., 27, 125-149.

Bowden M., and H. F. Eden, (1968) Effect of a radial barrier on the convective flow in a rotating fluid annulus, J. Geophys. Res., 73, 6887-6895.

Broomhead, D. S., and G. P. King (1986), Extracting qualitative dynamics from experimental data, Phys. D, 20, 217-236.

Cole, R. J. (1971), Hysteresis effects in a differentially heated rotating fluid annulus, Quart. J. R. Meteor. Soc., 97, 506-518.

DelSole, T. (2007), Optimal pertubations in quasigeostrophic turbulence, J. Atmos. Sci., 64, 1350-1364.

Dettinger, M. D., M. Ghil, C. M. Strong, W. Weibel, and P. Yiou (1995), Software expedites singular-spectrum analysis of noisy time series, Eos Trans. AGU, 76(2), 12, 14, 21.

Fasshauer, G. E. (2007), Meshless Approximation Methods with $M A T L A B$, Interdisciplinary Mathematical Sciences, Vol. 6, world Scientific Publishers, Singapore.

Fein, J. S. (1973), An experimental study of the effects of the upper boundary condition on the thermal convection in a rotating, differentially heated cylindrical annulus of water. Geophys. Fluid Dyn., 5, 213-243.

Foias, C., O. Manley, R. Rosa, and R. Temam (2008), NavierStokes Equations and Turbulence, Encyclopedia of Mathematics and Its Applications, Cambridge University Press, Cambridge.

Fowlis, W. W. and R. Hide (1965), Thermal convection in a rotating annulus of liquid: Effect of viscosity on the transition between axisymmetric and non-axisymmetric flow regimes, J. Atmos. Sci., 22, 541-558.

Früh, W.-G. (1996), Low-order models of wave interactions in the transition to baroclinic chaos. Nonlin. Process. Geophys., $3,150-165$. 
Früh, W.-G. and P. L. Read (1997), Wave interactions and the transition to chaos of baroclinic waves in a thermally driven rotating annulus, Phil. Trans. R. Soc. Lond. A, 355, 101-153.

Fultz, D. (1961), Development in controlled experiments on larger scale geophysical problems, Adv. Geophys., 1-104.

Fuselier, E. J. and G. B. Wright (2009), Stability and error estimates for vector field interpolation and decomposition on the sphere with RBFs, SIAM J. Num. Anal., 47, 3213-3239.

Gyüre, B., I. Bartos, and I. M. Jánosi (2007), Nonlinear statistics of daily temperature fluctuations reproduced in a laboratory experiment, Phys. Rev. E, 76, 037,301.

Harlander, U. (2005), A high latitude quasigeostrophic delta plane model derived from spherical geometry, Tellus, 57 A, 43-54.

Harlander, U., R. Faulwetter, K. Alexandrov, and C. Egbers (2009a), Estimating local instabilities from data with application to geophysical flows, Advances in Turbulence XII, Springer Proceedings in Physics, 132, 163-167.

Harlander, U., H. Ridderinkhof, M. W. Schouten, and W. P. M. De Ruijter (2009b), Long term observations of transport, eddies, and Rossby waves in the Mozambique Channel, J. Geophys. Res., 114, C02003, doi:10.1029/2008JC004846.

Harlander, U., Th. von Larcher, Y. Wang, and C. Egbers (2011), PIV- and LDV-measurements of baroclinic wave interactions in a thermally driven rotating annulus, Exp. Fluids, 51, 37-49, doi:10.1007/s00348-009-0792-5.

Harlander, U., J. Wenzel, K. Alexandrov, Y. Wang, and C. Egbers (2012a), Simultaneous PIV- and thermography-measurements of partially blocked flow in a heated rotating annulus. Exp. Fluids, 52, 1077-1085, doi:10.1007/s00348-011-1195-y.

Harlander, U., G. B. Wright, and C. Egbers (2012b), Reconstruction of the 3D flow field in a differentially heated rotating annulus by synchronized particle image velocimetry and infrared thermography measurements, 16th Int. Symp. on Appl. of Laser Techniques to Fluid Mech., Lisbon, Portugal, 9-12 July, 16:4.5.1.

Hasselmann, K. F. (1988), PIPS and POPS: The reduction of complex dynamical systems using principal interaction and oscillation patterns, J. Geophys. Res., 93, 11015-11021.

Hide, R. (1958), An experimental study of thermal convection in a rotating fluid, Phil. Trans. R. Soc. Lond. A, 250, 441-478.

Hide, R. and P. J. Mason (1970), Baroclinic waves in a rotating fluid subject to internal heating, Phil. Trans. Roy. Soc. Lond. $A, 268,201-232$.

Hide, R. (2010), A path of discovery in geophysical fluid dynamics, Astron. Geophys., 51, 4.16-4.23.

Hignett, P., A. A. White, R. D. Carter, W. D. Jackson, and R. M. Small (1985), A comparison of laboratory measurements and numerical simulations of baroclinic wave flows in a rotating cylindrical annulus, Q. J. Roy. Meteor. Soc., 111, $131-154$.

Holmes, P., J. L. Lumley, and G. Berkooz (1996), Turbulence, Coherent Structures, Dynamical Systems and Symmetry, Cambridge University Press, Cambridge.

Elsner J. B., and A. A. Tsonis (1996), Singular Spectrum Analysis: A New Tool in Time Series Analysis, Plenum, New York.

Kaiser, J. A. C. (1970), Rotating deep annulus convection 2. wave instabilities, vertical stratification and associated theories. Tellus, 22, 275-287.
Kalnay, E. (2002), Data Assimilation and Predictability. Cambridge University Press, Cambridge.

Lewis, G. M. and W. Nagata (2004), Linear stability analysis for the differentially heated rotating annulus, Geophys. Astrophys. Fluid Dyn., 98, 279-299.

Lindzen, R. S., B. Farrell, and D. Jacqmin (1982), Vacillation due to wave interference: Applications to the atmosphere and to annulus experiments, J. Atmos. Sci., 39, 14-23.

Lorenz, E. N. (1956), Empirical orthogonal functions and statistical weather prediction. Scientific Report No. 1, Statistical Forecasting Project. Air Force Research Laboratories, Office of Aerospace Research, USAF, Bedford, MA.

Lorenz, E. N. (1962), Simplified dynamic equations applied to the rotating-basin experiments. J. Atmos. Sci., 19, 39-51.

Lorenz, E. N. (1964), The problem of deducing the climate from the governing equations, Tellus, 16(1), 1-11.

Lorenz. E. N. (1984), Irregularity: A fundamental property of the atmosphere, Tellus, 36 A, 98-101.

Mason, P. J. (1975), Baroclinic waves in a container with sloping end walls. Phil. Trans. R. Soc. Lond. A, 278, 397-445.

Maxworthy, T., and F. K. Browand (1974), Experiments in rotating and stratified flows: Oceanographic application, Ann. Rev. Fluid Mech., 7, 273-305.

Miller, T. L., and R. L. Gall (1983), A linear analysis of the transition curve for the baroclinic annulus, J. Atmos. Sci., 40, 2293-2303.

Morita O., and M. Uryu (1989), Geostrophic turbulence in a rotating annulus of fluid, J. Atmos. Sci., 46, 2349-2355.

Mundt, M. D., and J. E. Hart (1994), Secondary instability, EOF reduction, and the transition to baroclinic chaos, Phys. D, 78, 65-92.

Narcowich F. J., and J. D. Ward (1994), Generalized Hermite interpolation via matrix-valued conditionally positive definite functions. Math. Comp., 63, 661-687.

Penland, C., and P. D. Sardeshmukh (1995), The optimal growth of sea surface anomalies, J. Clim., 8, 1999--2024.

Pfeffer, R. L., and W. W. Fowlis. (1968), Wave dispersion in a rotating differentially heated cylindrical annulus of fluids, J. Atmos. Sci., 25, 361-371.

Pfeffer, R. L., G. Buzyna, and R. Kung (1980), Time-dependent modes of thermally driven rotating fluids, J. Atmos. Sci., 37, 2129-2149.

Pfeffer, R. L., J. Ahlquist, R. J. Kung, Y. Chang, and G. Q. Li (1990), Study of baroclinic wave behavior over bottom topography using complex principal component analysis of experimental data. J. Atmos. Sci., 47, 67-81.

Pfeffer, R. L., S. R. Applequist, R. Kung, C. Long, and G. Buzyna (1997), Progress in characterizing the route to geostrophic turbulence and redesigning thermally driven rotating annulus, Theor. Comput. Fluid Dyn., 9, 253-267.

Pierrehumbert, R. T., and K. L., Swanson (1995), Baroclinic instability. Ann. Rev. Fluid Mech., 27, 419-467.

Randriamampianina, A., W.-G. Früh, P. L. Read, and P. Maubert (2006), Direct numerical simulations of bifurcations in an air-filled rotating baroclinic annulus, J. Fluid Mech., 561, 359-389.

Ravela, S., J. Marshall, C. Hill, A. Wong, and S. Stransky (2010), A realtime observatory for laboratory simulation of planetary flows, Exp. Fluids, 48, 915-925. 
Rayer, Q. G., D. W. Johnson, and R. Hide (1998), Thermal convection in a rotating fluid annulus blocked by a radial barrier. Geophys. Astrophys. Fluid Dyn., 87, 215-252.

Read, P. L. (1992), Applications of singular systems analysis to "baroclinic chaos". Phys. D, 58, 455-468.

Read, P. L., (1993), Phase portrait reconstruction using multivariate singular systems analysis, Phys. D, 69, 353-365.

Read, P. L., M. J. Bell, D. W. Johnson, and R.M. Small (1997), Quasi-periodic and chaotic flow regimes in a thermaly-driven, rotating fluid annulus, J. Fluid Mech., 238, 599-632.

Read, P. L., P. Maubert, A. Randriamampianina, and W.-G. Früh (2008), Direct numerical simulation of transitions towards structural vacillation in an air-filled, rotating, baroclinic annulus. Phys. Fluids., 20, 044107:1-17.

Rintoul, S. R., C. Hughes, and D. Olbers (2001), The Antarctic Circumpolar Current System, in Ocean Circulation and Climate, edited by G. Siedler, J. Gould, and J. Church, Elsevier Science, New York.

Scolan, H., J.-B. Flor, R. Verzicco. Frontal instabilities at a density-shear interface in rotating two-layer stratified fluid, chapter 11, this issue.

Seelig, T., U. Harlander, R. Faulwetter, and C. Egbers (2012), Irregularity and singular vector growth in the differentially heated rotating annulus, Theor. Comp. Fluid Dyn., doi:10.1007/s00162-011-0255-5.

Sitte, B., and C. Egbers (2000), Higher order dynamics of baroclinc waves. in Physics of Rotating Fluids, edited by G. Pfister and, C. Egbers, pp. 355-375, Springer, Berlin.

Stein, M. L. (1999), Interpolation of Spatial Data: Some Theory for Kriging, Springer Series in Statistics, Springer-Verlag, New York.

Stephen, A. V., I. M. Moroz, P. L. Read, and W.-G. Früh (1997), A comparison of empirical orthogonal decomposition methods in baroclinic flows, Dyn. Atmos. Oceans, 27, 649-660.

Stephen, A. V., I. M. Moroz, and P. L. Read (1999), POD analysis of baroclinic wave flows in the thermally-driven, rotating annulus experiment, Phys. Chem. Earth (B), 24, 449-453.

Trefethen, L. N., A. E. Trefethen, S. C. Reddy, and T. A. Driscoll (1993), On almost rigid rotations. part 2, Science, 261, 578-584.

Vautard, R. (1995), Patterns in time: SSA and MSSA, in Analysis of Climate Variability, edited by $\mathrm{H}$. Von Storch, and A. Navarra, Springer, Berlin.

von Larcher, Th., and C. Egbers (2005a), Dynamics of baroclinic instabilities using methods of nonlinear time series analysis, in
J. Peinke, A. Kittel, S. Barth, and M. Oberlack, edited by Progress in Turbulence, edited by Springer, Berlin.

Von Larcher, Th., and C. Egbers (2005b), Experiments on transitions of baroclinic waves in a differentially heated rotating annulus, Nonlin. Proc. Geophys., 12, 1033-1041.

von Larcher, Th., A. Fournier, and R. Hollerbach (2013), The influence of a sloping bottom endwall on the linear stability in the thermally driven rotating annulus with a free surface, Theoretical and Computational Fluid Dynamics, 27(3), 433-451.

von Larcher, Th. and A. Dörnbrack, Numerical simulations of baroclinic driven flows in a thermally driven rotating annulus using the immersed boundary method, Meteorologische Zeitschrift, to appear.

Von Storch, H., (1995), Statistical patterns: EOFs and CCA, in Analysis of climate variability, edited by $\mathrm{H}$. Von Storch, and A. Navarra, Springer, Berlin.

Von Storch, H., and F. W. Zwiers (1999), Statistical analysis in climate research, by Storch, $\mathrm{H}$. von and Zwiers, F.W. Cambridge University Press, Cambridge, 1999, No. of Pages x +484pp. Price US\$65-00, ISBN 0521450713 (Hardback).

J.-S. von Storch (1995), Multivariate statistical modeling: POPmodel as a first order approximation. in Analysis of climate variability, edited by H. von Storch and A. Navarra, Springer, Berlin.

Weeks, E. R., Y. Tian, J. S. Urbach, K. Ide, H.L. Swinney, and M. Ghil (1997), Transitions between blocked and zonal flows in a rotating annulus with topography. Science, 278, 1598-1601.

Weng, H.-Y., A. Barcilon, and J. Magnan (1986), Transitions between baroclinic flow regimes, J. Atmos. Sci., 43, 1760-1777.

Wilson, C., and R. G. Williams (2006), When are eddy tracer fluxes directed downgradient? J. Phys. Oceanogr., 36, 189-201.

Will, A., U. Harlander, and W. Metz (2006), Climatological relevance of leading seasonal singular vectors. Part I: Energy, enstrophy and spatiotemporal variability, Meteorologische Zeitschrift, 15, 463-472.

Williams, P. D., T. W. N. Haine, and P. L. Read (2008), Inertiagravity waves emitted from balanced flow: Observations, properties and consequences. J. Atmos. Sci., 65, 3543-3556.

Yang, H., (1990), Wave Packets and Their Bifurcations in Geophysical Fluid Dynamics. Springer Verlag, New York.

Young, R. M. B., and P.L. Read (2008), Breeding and predictability in the baroclinic rotating annulus using a perfect model, Nonlin. Processes Geophys., 15, 469-487. 